\title{
Comparative Analysis of the Usefulness of AIS and ARPA for Anti-collision Purposes
}

\author{
R. Wawruch \\ Gdynia Maritime University, Gdynia, Poland
}

ABSTRACT: The article discusses the principles of presenting data available from AIS and radar tracking on ship's radar display units and describes the results of comparative studies of the accuracy of their indications on sea-going vessels in real meeting situations in various hydro meteorological conditions.

\section{INTRODUCTION}

In accordance with the current international recommendations set out in the Resolution MSC.192(79) "Adoption of the revised performance standards for radar equipment" adopted by the International Maritime Organisation (IMO) on 6th of December 2004, each radar installed on or after 1st of July 2008 on ships subject to the requirements of SOLAS regulation V / 19 should present data from radar tracking and on board automatic identification system (AIS) [4]. Radar tracking aids (automatic radar plotting aid - ARPA and automatic tracking aid ATA) calculate true vector, the passing distance with detected and tracked object and time to pass it, called respectively closest point of approach (CPA) and time to the closest point of approach (TCPA) on the basis of radar measurements of its distance and bearing. AIS at given time intervals and in pulling mode automatically transmits, receives and displays, inter alia, indications of connected to it gyrocompass, speed and distance measuring device and Global Navigational Satellite System (GNSS), currently mainly GPS, ship's receiver. Additionally, it can calculate and indicate CPA and TCPA on the basis of knowledge of the geographical positions and true motion vectors of the own ship and the opposite vessel transmitting AIS messages. Relatively low accuracy of radar measurements means that the data from radar tracking can be presented with lower accuracy than data from AIS. Due to that, when the target data from AIS and radar tracking are both available and the association criteria (position, motion, etc.) are fulfilled, i.e. the AIS and radar information is considered as concerning one physical target, then the AIS target symbol and the alphanumerical AIS target data should be automatically selected and presented on the radar display as a default condition [4].

However, the radar remains the only independent technical source of information about surface objects around own ship. For this reason, according to the recommendations of the Resolution A.1106(29) "Revised guidelines for the operational use of shipborne automatic identification systems (AIS)" adopted by IMO on 2nd of December 2015, the AIS may be recommended as an anti-collision device in due time and its introduction has not impact on the Rule 19 "Conduct of vessels in restricted visibility" of the International Regulations for Preventing Collisions at Sea (COLREG) and its interpretation. The ship's master and watch keeping officers (OOW) should not rely on AIS as the sole information system, 
but should make use of all safety-relevant information available. In general, AIS may be used to assist in collision avoidance decision-making as an additional source of information which supports radar and radar tracking aids to calculate true and relative vectors of the detected and tracked echoes on the basis of radar measurements of their distances and bearings, by assisting in $[3,13]$ :

- identification of targets by name, call sign, ship type and navigational status;

- presentation of targets heading;

- immediate identification of manoeuvres performed by targets; and

- more accurate presentation of courses, speed over ground and rate of turn of the targets.

IEC Standard 61993-2 presenting performance standards for AIS required that if AIS display equipment provides facilities for the calculation of CPA and TCPA then these facilities should comply with the relevant clauses of the IEC Standard 62388 "Shipborne radar - Performance requirements, methods of testing and required test results" [1]. The said standard specifies the minimal requirements for radar conforming to performance standards not inferior to those adopted by IMO in the Resolution MSC.192(79) [2]. According to both standards, accuracy of radar tracking shall be as presented in Table $1[2,4]$. Mentioned in this table time of steady state tracking means radar tracking a target in the steady phase of movement $[2,4]$ :

- after completion of the acquisition process; or

- without a manoeuvre of target or own ship; or

- without target swap or any disturbance.

In maritime navigation nautical miles (NM) and knots $(\mathrm{kn})$ are officially used as the units of distance and speed. ARPA and AIS present values of distance, CPA and speed in these units. Therefore, in this paper they are presented in nautical miles and kilometres and in knots and m/s $(1 \mathrm{NM}=1852 \mathrm{~m} ; 1 \mathrm{kn}=1 \mathrm{NM} / \mathrm{h}$ $\approx 0.514 \mathrm{~m} / \mathrm{s}$ ) respectively.

Resolution MSC.192(79) informs additionally that automatic tracking accuracy [4]:

- should be achieved assuming the sensor errors allowed by the relevant IMO performance standards (range and bearing accuracy should be within $50 \mathrm{~m}$ (or $+/-1 \%$ of target range) and $2^{\circ}$ ); and

- may be significantly reduced during or shortly after acquisition, own ship manoeuvre, a manoeuvre of the target, or any tracking disturbance and is also dependent on own ship's motion and sensor accuracy.

The testing standard should comprise detailed target simulation tests as a means to confirm the accuracy of targets with relative speeds of up to 100 $\mathrm{kn}$. The operating instructions should contain a qualified explanation and/or description of information required by the user to operate the radar system correctly, including limitations of the display and tracking process and accuracy, including any delays [4].

The Standard IEC 62388 requires that the tracking accuracy be checked using simulated targets generated by a target simulator in a noise-free and clutter-free environment. Table 1 provides an indication of typical tracking accuracy, averaged over five tracking scenarios and with minimal sensor errors as described in Annex $E$ to that standard. The individual scenarios are as follows [2]:

- scenario 1 applies the sensor errors as defined in Annex E in this standard;

- scenarios 2 and 3 test own ship turns in both directions, without sensor errors;

- scenario 4 tests for target swap, without sensor errors; and

- scenario 5 provides 10 targets, including one having a $50 \%$ visibility; no sensor errors are applied.

The test scenarios differentiate standard and high speed craft by the parameters used. They simulate own ship travelling at up to $30 \mathrm{kn}$ (or up to $70 \mathrm{kn}$ for high speed craft), whilst tracking targets with a speed of up to $70 \mathrm{kn}$. High rate of turn, own ship and target manoeuvring, target swap, multiple targets on a bearing, acceleration and fading are simulated. The simulator assumes a $2.0^{\circ}$ antenna ( $-3 \mathrm{~dB}$ point) horizontal beam width, an antenna rotation rate compatible with the category of equipment, and at a pulse length and pulse repetition frequency as specified by the manufacturer [2].

Additionally, the tracking system shall demonstrate tracking capability in noisy and clutter environments. This requirement shall be checked by visual observation. It is fulfilled if the observer confirms that when [2]:

- the test targets in scenario 5 are set to $10 \mathrm{~dB}$ above peak noise level, they are tracked without degradation of the tracking performance; and

- the tracking system is operated in a typical clutter environment and using targets of opportunity (targets of different sizes, speeds and trajectories), continue to be tracked with minimal degradation to tracking performance.

All of the information provided shows that presented in Table 1 radar tracking accuracy and accuracy of the CPA and TCPA values presented by AIS apply only to conditions simulated in the absence of noise and clutters. The issue requiring research is the accuracy of the analyzed indications on seagoing ships of different sizes in real conditions of their exploitation and in real meeting situations.

The measurements reported in this article were carried out to determine;

- the mean values and mean square errors of data presented by AIS and ARPA;

- the influence of the stability of the observed and tracked ship's motion on the accuracy of determining its true motion vector by ARPA and CPA by ARPA and AIS as a function of time; and

- the relationship between the accuracy of AIS and ARPA indications and the current state of the sea.

They were carried out over several years and some of their results have already been partly presented in publications [12-15]. 
Table 1. Tracked target accuracy (95\% probability figures) $[2,4]$

\begin{tabular}{|c|c|c|c|c|c|c|}
\hline $\begin{array}{l}\text { Time of steady } \\
\text { state tracking } \\
\text { [min] }\end{array}$ & $\begin{array}{l}\text { Relative course } \\
{\left[{ }^{\circ}\right]}\end{array}$ & $\begin{array}{l}\text { Relative speed } \\
{[\mathrm{kn} / \mathrm{m} / \mathrm{s}]}\end{array}$ & $\begin{array}{l}\text { CPA } \\
{[\mathrm{NM} / \mathrm{km}]}\end{array}$ & $\begin{array}{l}\text { TCPA } \\
{[\mathrm{min}]}\end{array}$ & $\begin{array}{l}\text { True course } \\
{\left[{ }^{\circ}\right]}\end{array}$ & $\begin{array}{l}\text { True speed } \\
{[\mathrm{kn} / \mathrm{m} / \mathrm{s}]}\end{array}$ \\
\hline $1 \mathrm{~min}$ : trend & 11 & $\begin{array}{l}1.5 / 0.8 \text { or } 10 \% \\
\text { (whichever is greater) }\end{array}$ & $1.0 / 1.85$ & - & - & - \\
\hline 3 min: motion & 3 & $\begin{array}{l}0.8 / 0.4 \text { or } 1 \% \\
\text { (whichever is greater) }\end{array}$ & $0.3 / 0.56$ & 0.5 & 5 & $\begin{array}{l}0.5 / 0.3 \text { or } 1 \% \\
\text { (whichever is greater) }\end{array}$ \\
\hline
\end{tabular}

Table 2. Ships on which tests were carried out with the indication of their size category and on board AIS and radar equipment $[5,6,7,8,9,10,11,16,17,18]$

\begin{tabular}{|c|c|c|c|c|c|c|}
\hline & Ship & & & & Used equipment / manufacturer & \\
\hline Size & Type & $\begin{array}{l}\text { Gross } \\
\text { tonnage }\end{array}$ & $\begin{array}{l}\text { Length } \\
{[\mathrm{m}]}\end{array}$ & $\begin{array}{l}\text { Service speed } \\
{[\mathrm{kn} / \mathrm{m} / \mathrm{s}]}\end{array}$ & Radar / ARPA & AIS \\
\hline $\mathrm{V}$ & Bulk carrier & 106884 & 299.91 & $5.6 / 8.0$ & JMA-9132-SA, JMA-9122-9XA / JRC & JHS-183 / JRC \\
\hline $\mathrm{L}$ & LPG tanker & 46789 & 226.0 & $16.7 / 8.6$ & JMA-9172-SA, JMA-9122-9XA / JRC & JHS-183 / JRC \\
\hline $\mathrm{L}$ & Multipurpose ship & 30469 & 199.8 & $16.8 / 8.6$ & $\begin{array}{l}\text { Radar Vision Master FT X, S bands, } \\
\text { ARPA 340/25X, 340/30S / Sperry Marine }\end{array}$ & $\begin{array}{l}\text { Nauticast X-Pack } \\
\text { DS / Nauticast GmbH }\end{array}$ \\
\hline M & Multipurpose ship & 11864 & 143.0 & $13.2 / 6.8$ & $\begin{array}{l}\text { GR3017 (X-Band), GR3018 (S-Band), } \\
\text { ARPA Multipilot } 1100 \text { / SAM } \\
\text { Electronics GmbH }\end{array}$ & $\begin{array}{l}\text { DEBEG } 3400 \text { / SAM } \\
\text { Electronics GmbH }\end{array}$ \\
\hline M & Liqufied gas c & r 22941 & 174.2 & $15.5 / 8.0$ & JMA-9932-SA, JMA-9922-6X / JRC & JHS-182 / JRC \\
\hline M & Bulk carrier & 20603 & 190 & $14.0 / 7.2$ & FAR-21X7(-BB) (X \& S BAND), Furuno & FA-150 / Furuno \\
\hline M & Container & 16801 & 184.1 & $19.7 / 10.1$ & $\begin{array}{l}\text { X \& S Sperry Marine / Northtop } \\
\text { Grumman }\end{array}$ & R4 / SAAB \\
\hline S & Bulk carrier & 2735 & 89.4 & $11.0 / 5.7$ & FAR-28x7 model FAR-21x7 (-BB) / Furuno & $\begin{array}{l}\text { R4 / SAAB SAAB } \\
\text { Transponder Tech }\end{array}$ \\
\hline $\begin{array}{l}\mathrm{AB} \\
\mathrm{S}\end{array}$ & Oil/chemical tanker & r 4667 & 97.4 & $13.6 / 6.7$ & Vision Master FT CAT 2 / Sperry Marine & $\begin{array}{l}\text { R4 / SAAB } \\
\text { Transponder Tech }\end{array}$ \\
\hline $\begin{array}{l}\mathrm{AB} \\
\mathrm{S}\end{array}$ & General cargo & 3443 & 93.42 & $12.0 / 6.2$ & Bridge Master E (S) / Sperry Marine & JHS-182/ JRC \\
\hline
\end{tabular}

V- very large; L - large; M - medium; S - small

\section{DESCRIPTION OF THE MEASUREMENTS}

The measurements were conducted by students of the Faculty of Navigation of the Gdynia Maritime University as part of their engineering thesis listed in bibliography $[5,6,7,8,9,10,11,16,17,18]$, written under the supervision of the author of this article. They were done in real (not simulated) conditions during the sea voyages of ten different ships presented in Table 2, using AIS and radar equipment installed on these vessels and mentioned in this table too. The division of vessels into size categories was made conventionally by the author of the paper. For the analyses presented in this article, only measurement series were selected, during which both the own ship and the tracked vessel were proceeding with a steady course and constant speed without performing any manoeuvres. Any instabilities and errors of CPA and true vector indications were therefore caused only by inaccuracies in radar measurements and the influence of the current hydro meteorological conditions, mainly sea state, on the ship's movement.

Table 3 presents information on all ships tracked during measurements, their names, type, length, speed and distance to the own ship. They are grouped according to the type of meeting situation with own ship (parallel courses - overtaking, reciprocal courses and crossing courses) and to the size of the vessel from which the measurements were taken. The results of measurements during which both ships (own and observed) were at anchors are presented separately at the end of the Table 3. The state of the sea, expressed in degrees of the Douglas scale in the last column of this table, describes weather conditions during the test. Part of the measurements presented in this table is a repetition of information presented in the earlier publications mentioned in the bibliography [12-15].

During each test were recorded, simultaneously every 30 seconds, following parameters of the observed vessel indicated by AIS and ARPA: true bearing, distance, true course, true speed, CPA and time to reach CPA (TCPA). In order to meet the conditions of steady state tracking set out in the IMO resolution and the IEC standard, observed ships were tracked by ARPAs for at least 5 minutes before the beginning of registration and both vessels (own and opposite) did not take any manoeuvres at this time and later during the registration.

The terms and abbreviations used in Table 3 mean:

- Distance - distance between the ships (own and observed) during the measurement;

- L - the length of the observed vessel presented on the web-site;

- No/size category - consecutive number of the measurement series / ship size category;

- T - type of the ship indicated by AIS: B - bulk carrier, C - container vessel, CS - cargo ship, D dredger, F - ferry boat, FV - fishing vessel, P - 
passenger ship, RO - ro-ro vessel, SP - special - Sea state - state of the sea expressed in degrees of purpose ship, $\mathrm{T}$ - tanker; and the Douglas scale, sw means swell.

Table 3. Ships observed during tests divided according to the meeting situation and size category of the vessel from which the measurements were conducted [5-18]

\begin{tabular}{|c|c|c|c|c|c|c|}
\hline $\begin{array}{l}\text { No/size } \\
\text { category }\end{array}$ & Ship's name & $\mathrm{T}$ & $\mathrm{L}[\mathrm{m}]$ & Speed $[\mathrm{kn} / \mathrm{m} / \mathrm{s}]$ & Distance $[\mathrm{M} / \mathrm{km}]$ & $\begin{array}{c}\text { Sea } \\
\text { state }\end{array}$ \\
\hline \multicolumn{7}{|c|}{ Parallel courses - overtaking } \\
\hline $1 / \mathrm{V}$ & Belgian Express & $\mathrm{C}$ & 180 & $13.0 / 6.7$ & $1.0-0.7 / 1.9-1.3$ & 4 \\
\hline $2 / \mathrm{V}$ & China Peace & $\mathrm{B}$ & 289 & $0 / 0$ & $16.8-14.5$ / 31.1-26.9 & 3 \\
\hline $3 / \mathrm{V}$ & Lena River & $\mathrm{T}$ & 290 & $0 / 0$ & 6.7-3.4 / 12.4-6.3 & 2 \\
\hline $4 / \mathrm{V}$ & Ocean Trader & CS & 180 & $11.1 / 5.7$ & 19.8-19.6 / 36.7-36.3 & 4 \\
\hline $5 / \mathrm{V}$ & Regio Mar & FV & 21 & $8.0 / 4.1$ & $6.5-3.0 / 12.0-5.6$ & 2 \\
\hline $6 / \mathrm{V}$ & Tian Zhu Feng & B & 225 & $10.8 / 5.6$ & $16.8-16.4$ / 31.1-30.4 & 7 \\
\hline $7 / \mathrm{L}$ & F.D. Gennaro Aurilia & B & 225 & $12.0 / 6.2$ & $14.1-13.2$ / 26.1-24.4 & 3 \\
\hline $8 / \mathrm{L}$ & Hyundai Unity & C & 294 & $13.3 / 6.8$ & $9.3 / 17.2$ & 3 \\
\hline 9/L & Suez Vasilis & $\mathrm{T}$ & 274 & $14.0 / 7.2$ & $2.0-1.8 / 3.7-3.3$ & 4 \\
\hline $10 / \mathrm{L}$ & Thorco Raffles & CS & 161.5 & $11.8 / 6.1$ & 6.8-6.7 / 12.6-12.4 & 3 \\
\hline $11 / \mathrm{L}$ & Creole Spirit & $\mathrm{T}$ & 295 & $11.5 / 5.9$ & $12.5-10.6$ / 23.2-19.6 & 2 \\
\hline $12 / \mathrm{L}$ & Lake Kivu & CS & 182 & $15.5 / 8.0$ & 8.7-8.2 / 16.1-15.2 & 2 \\
\hline $13 / \mathrm{M}$ & Alexandra & CS & 270 & $17.5 / 9.0$ & $2.5-2.2$ / 4.6-4.1 & 2 \\
\hline $14 / \mathrm{M}$ & Celtic Ambasador & CS & 88 & $9.1 / 4.7$ & $13.4-12.4$ / 24.8-23.0 & 5 \\
\hline $15 / \mathrm{M}$ & Christopher & CS & 171 & $15.1 / 7.8$ & 1.8-1.6 / 3.3-3.0 & 5 \\
\hline $16 / \mathrm{M}$ & Coral Meandra & $\mathrm{T}$ & 91 & $11.1 / 5.7$ & $1.5-1.4 / 2.8-2.6$ & 4 \\
\hline $17 / \mathrm{M}$ & Corcovado & CS & 207 & $0.5 / 0.3$ & 18.9-15.8 / 35.0-29.3 & 1 \\
\hline $18 / \mathrm{M}$ & CSCL Jupiter & CS & 366 & $18.2 / 9.4$ & 3.6-2.6 / 6.7-4.8 & 3 \\
\hline $19 / \mathrm{M}$ & Flinter Aland & CS & 132 & $10.7 / 5.5$ & 7.9-7.1 / 14.6-13.1 & 4 \\
\hline $20 / \mathrm{M}$ & Heinrich & $\mathrm{T}$ & 114 & $11.9 / 6.1$ & 18.6-18.1 / 34.4-33.5 & 2 \\
\hline $21 / \mathrm{M}$ & Histria Ivory & $\mathrm{T}$ & 179 & $10.8 / 5.6$ & $17.1-17.0$ / 31.7-31.5 & 6 \\
\hline $22 / \mathrm{M}$ & Navin Kestrel & CS & 116 & $10.2 / 5.3$ & $5.2-4.4$ / 9.6-8.1 & 3 \\
\hline 23/M & Pacific Heron & SP & 88 & $4.8 / 2.5$ & $9.0-7.8$ / 16.7-14.4 & 1 \\
\hline $24 / \mathrm{M}$ & Panther & CS & 207 & $16.1 / 8.3$ & $11.4-10.9$ / 21.1-20.2 & 3 \\
\hline 25/M & Union Ranger & CS & 185 & $11.7 / 6.0$ & 18.7-18.4 / 34.6-34.1 & 1 \\
\hline $26 / \mathrm{M}$ & Varvara & CS & 225 & $11.2 / 5.8$ & 5.7-5.1 / 10.6-9.4 & 3 \\
\hline $27 / \mathrm{M}$ & MSC India & CS & 278 & $18.0 / 9.3$ & $4.4-3.6$ / 8.2-6.7 & 2 \\
\hline $28 / \mathrm{M}$ & Grande Cotonou & CS & 236 & $14.6 / 7.5$ & $15.0-14.8$ / 27.8-27.4 & 2 \\
\hline 29/M & Lacerta & $\mathrm{T}$ & 183 & $14.2 / 7.3$ & 6.7-6.1 / 12.4-11.3 & 3 \\
\hline $30 / \mathrm{M}$ & Anne & $\mathrm{T}$ & 333 & $0.5 / 0.3$ & $17.8-14.8$ / 33.0-27.4 & 3 \\
\hline $31 / \mathrm{M}$ & Mosvik & CS & 82 & $5.7 / 2.9$ & $1.2-0.7 / 2.2-1.3$ & 5 \\
\hline $32 / \mathrm{M}$ & SFC Don & $\mathrm{T}$ & 183 & 11.9 / 6.1 & $1.0-0.9$ / 1.9-1.7 & 5 \\
\hline $33 / \mathrm{M}$ & Nordica Hav & CS & 83 & 6.2 / 3.2 & $1.8-0.9 / 3.3-1.7$ & 5 \\
\hline $34 / \mathrm{M}$ & Sca Ortviken & $\mathrm{RO}$ & 170 & $13.6 / 7.0$ & $1.1-0.5 / 2.0-0.9$ & 4 \\
\hline $35 / \mathrm{M}$ & Iron Kovdor & $\mathrm{B}$ & 225 & $11.3 / 5.8$ & $4.1-3.9 / 7.6-7.2$ & 4 \\
\hline $36 / \mathrm{M}$ & Alexandra & FV & 24 & $2.8 / 1.4$ & $4.8-3.8$ / 8.9-7.0 & 2 \\
\hline $37 / \mathrm{M}$ & Shiosai & B & 289 & $9.0 / 4.6$ & 9.4-3.5 / 17.4-6.5 & 5 \\
\hline $38 / \mathrm{M}$ & Maersk Columbus & C & 299 & $17.0 / 8.7$ & 20.4-18.9 / 37.8-35.0 & 1 \\
\hline $39 / \mathrm{M}$ & Iver Bitumen & $\mathrm{T}$ & 109 & $4.8 / 2.5$ & $3.3-3.0 / 6.1-5.6$ & 7 \\
\hline $40 / \mathrm{M}$ & Oceana & $\mathrm{P}$ & 261 & $18.2 / 9.4$ & $24.4-23.8$ / 45.2-44.1 & 1 \\
\hline $41 / \mathrm{M}$ & Fuji Bay & CS & 148 & $16.7 / 8.6$ & 17.7-14.1 / 32.8-26.1 & 1 \\
\hline $42 / S$ & Britannia & $\mathrm{P}$ & 329 & $17.0 / 8.7$ & $4.1-2.2$ / 7.6-4.1 & 1 \\
\hline $43 / S$ & Finnstar & $\mathrm{P}$ & 219 & $22.7 / 11.7$ & 6.4-0.5 / 11.9-0.9 & 4 \\
\hline $44 / S$ & Gilingham & CS & 190 & 13.0 / 6.7 & $5.7-4.7 / 10.6-8.7$ & 4 \\
\hline $45 / S$ & Hafnia Sea & CS & 187 & $18.5 / 9.5$ & $6.0-2.6 / 11.1-4.8$ & 2 \\
\hline $46 / S$ & Kompozitor Rakhmaninov & CS & 126 & $9.6 / 4.9$ & $2.2-2.1 / 4.0-3.9$ & 1 \\
\hline $47 / S$ & Navi Star & CS & 110 & $11.5 / 5.9$ & $1.8-1.7 / 3.3-3.1$ & 1 \\
\hline $48 / S$ & Sea Explorer & CS & 110 & $11.5 / 5.9$ & $0.9-0.8 / 1.7-1.5$ & 2 \\
\hline $49 / S$ & Beaumonde & CS & 89 & $4.4 / 2.3$ & 5.8-5.7 / 10.7-10.6 & 3 \\
\hline $50 / S$ & Ogino Park & CS & 145 & $12.2 / 6.3$ & $4.8-4.5 / 8.9-8.3$ & 3 \\
\hline $51 / S$ & SSI Pride & CS & 190 & $10.5 / 5.4$ & $4.2-4.1 / 7.8-7.6$ & 6 \\
\hline $52 / S$ & Pinnau & CS & 88 & $10 / 5.1$ & $2.8-2.7 / 5.2-5.0$ & 3 \\
\hline $53 / S$ & John Friedrich $\mathrm{K}$ & CS & 89 & $10.5 / 5.4$ & $1.1-0.7 / 2.0-1.3$ & 2 \\
\hline $54 / S$ & Abidjan EXpress & CS & 222 & $18.5 / 9.5$ & $5.2-5.0 / 9.6-9.3$ & 3 \\
\hline $55 / S$ & Muflao & $\mathrm{FV}$ & 16 & $0.9 / 0.5$ & $3.5-2.0 / 6.5-3.7$ & 2 \\
\hline $56 / S$ & Varkan Marmara & CS & 100 & $5.7 / 2.9$ & $1.1-0.7 / 2.0-1.3$ & 5 \\
\hline $57 / S$ & Aldebaran & CS & 90 & $9.2 / 4.7$ & $1.0-0,7 / 1.8-1.3$ & 2 \\
\hline $58 / S$ & Belmar & $\mathrm{T}$ & 249 & $5.0 / 2.6$ & $4.1-2.8 / 7.6-5.2$ & 3 \\
\hline $59 / S$ & Fortuna & CS & 87 & $9.0 / 4.6$ & $0.7-0.5 / 1.3-0.9$ & 3 \\
\hline $60 / S$ & Maersk Privilege & $\mathrm{T}$ & 240 & $1.6 / 0.8$ & 4.1-2.1 / 7.6-3.9 & 4 \\
\hline $61 / S$ & Stone & Tug & 37 & $7.8 / 4.0$ & $1.1-0.5 / 2.0-0.9$ & 3 \\
\hline \multicolumn{7}{|c|}{ Reciprocal courses } \\
\hline $62 / \mathrm{V}$ & APL Vancuver & $\mathrm{C}$ & 328 & $19.2 / 9.9$ & 18.4-13.1 / 34.1-24.3 & 4 \\
\hline $63 / \mathrm{V}$ & Jacamar Arrow & B & 199 & $14.0 / 7.2$ & 5.5-1.3 / 10.2-2.4 & 5 \\
\hline $64 / \mathrm{L}$ & Cosco Jinggangshan & B & 177 & $10.0 / 5.1$ & 13.1-6.6 / 24.3-12.2 & 5 \\
\hline $65 / \mathrm{L}$ & HSC & B & 289 & $11.6 / 6.0$ & 7.6-5.7 / 14.1-10.6 & 3 \\
\hline
\end{tabular}




\begin{tabular}{|c|c|c|c|c|c|c|}
\hline $66 / \mathrm{L}$ & Maersk Cape Coast & $\mathrm{C}$ & 249 & $15.0 / 7.7$ & $6.5-2.6 / 12.0-4.8$ & 1 \\
\hline $67 / \mathrm{L}$ & NYK Altair & $\mathrm{C}$ & 333 & $14.1 / 7.3$ & $12.5 / 23.2$ & 4 \\
\hline $68 / \mathrm{L}$ & Port Shanghai & B & 190 & $10.0 / 5.1$ & 7.7-2.5 / 14.3-4.6 & 5 \\
\hline $69 / \mathrm{L}$ & Varamo & $\mathrm{C}$ & 166 & $25.3 / 13.0$ & $6.9-1.2 / 12.8-2.2$ & 4 \\
\hline 70/L & Kota Buana & CS & 180 & $11.1 / 5.7$ & $11.6-5.3$ / 21.5-9.8 & 3 \\
\hline $71 / \mathrm{L}$ & Militos & $\mathrm{T}$ & 274 & $12.4 / 6.4$ & $5.4-2.7 / 10.0-5.0$ & 3 \\
\hline $72 / \mathrm{M}$ & Ara Antwerpen & CS & 145 & $11.2 / 5.8$ & $9.2-3.0 / 17.0-5.6$ & 4 \\
\hline $73 / \mathrm{M}$ & Beatriz B & CS & 159 & 12.9 / 6.6 & $19.0-12.9$ / 35.2-24.0 & 6 \\
\hline $74 / \mathrm{M}$ & Bomar Resolute & CS & 232 & $15.4 / 7.9$ & $7.0-1.8 / 13.0-3.3$ & 1 \\
\hline $75 / \mathrm{M}$ & Eken & $\mathrm{T}$ & 135 & 12 / 6,2 & $4.7-2.0 / 8.7-3.7$ & 2 \\
\hline $76 / \mathrm{M}$ & Gas Pasha & CS & 96 & $9.3 / 4.8$ & 14.1-8.4 / 26.1-15.6 & 3 \\
\hline $77 / \mathrm{M}$ & Hoegh Shanghai & CS & 229 & $9.2 / 4.7$ & 17.1-11.3 / 31.7-20.9 & 2 \\
\hline $78 / \mathrm{M}$ & Rome Trader & CS & 179 & $14.4 / 7.4$ & 19.5-12.6 / 36.1-23.3 & 2 \\
\hline $79 / \mathrm{M}$ & Rome Trader & CS & 179 & $14.4 / 7.4$ & 10.1-3.4 / 18.7-6.3 & 2 \\
\hline $80 / \mathrm{M}$ & Thorco Legion & CS & 132 & $12.35 / 6.4$ & $13.5-6.8$ / 25.0-12.6 & 3 \\
\hline $81 / \mathrm{M}$ & Cape Esmeralda & CS & 127 & $11.3 / 5.8$ & $14.2-10.6$ / 26.3-19.6 & 3 \\
\hline $82 / \mathrm{M}$ & Capella & $\mathrm{T}$ & 185 & $13.2 / 6.8$ & $4.4-3.0 / 8.1-5.6$ & 3 \\
\hline $83 / \mathrm{M}$ & Stena Germanica & $\mathrm{F}$ & 240 & 16.6 / 8.5 & 7.4-0.9 / 13.7-1.7 & 5 \\
\hline $84 / \mathrm{M}$ & Minerva Lisa & $\mathrm{T}$ & 243 & 13.4 / 6.9 & 7.5-1.3 / 13.9-2.4 & 5 \\
\hline $85 / \mathrm{M}$ & Arklov Brave & CS & 120 & $12.3 / 6.3$ & $6.7-4.6$ / 12.4-8.5 & 4 \\
\hline $86 / \mathrm{M}$ & Jing Lu Hai & B & 225 & 12.5 / 6.4 & $5.7-4.3$ / 10.6-8.0 & 4 \\
\hline 87/M & Hong Yu & B & 225 & $10.9 / 5.6$ & 6.0-0.9 / 11.1-1.7 & 6 \\
\hline $88 / \mathrm{M}$ & Luzon Strait & C & 167 & $20.5 / 10.5$ & $9.1-1.8$ / 16.9-3.3 & 4 \\
\hline $89 / \mathrm{M}$ & Aknoul & $\mathrm{RO}$ & 122 & $13.5 / 6.9$ & $6.2-4.6 / 11.5-8.5$ & 1 \\
\hline 90/M & LPG Barouda & $\mathrm{T}$ & 115 & $9.8 / 5.0$ & $11.0-4.2$ / 20.4-7.8 & 2 \\
\hline 91/M & Reggedijk & CS & 90 & $10.0 / 5.1$ & 9.3-7.4 / 17.2-13.7 & 2 \\
\hline 92/S & Baltic Advance & $\mathrm{T}$ & 182 & $11.1 / 5.7$ & $10.9-1.4$ / 20.2-2.6 & 3 \\
\hline $93 / S$ & Genco Thunder & CS & 225 & $10.2 / 5.2$ & 10.3-3.5 / 19.1-6.5 & 4 \\
\hline $94 / \mathrm{S}$ & Seabourn Ovation & $\mathrm{P}$ & 211 & 16.9 / 8.7 & $11.0-4.9$ / 20.4-9.1 & 2 \\
\hline 95/S & Tidan & CS & 88 & $10.5 / 5.4$ & $9.8-1.8$ / 18.1-3.3 & 2 \\
\hline $96 / S$ & Clipper Point & CS & 142 & $17.0 / 8.7$ & 10.6-3.3 / 19.6-6.1 & 5 \\
\hline $97 / S$ & Emek S & CS & 112 & 14.2 / 7.3 & 9.1-5.3 / 16.9-9.8 & 2 \\
\hline $98 / S$ & Sider Amy & CS & 136 & $13.1 / 6.7$ & 7.5-4.7 / 13.9-12.4 & 4 \\
\hline $99 / S$ & Tramaro Paris & CS & 154 & 12.2 / 6.3 & 7.7-4.6 / 14.3-8.5 & 6 \\
\hline $100 / S$ & Drenec & CS & 336 & $12.3 / 6.3$ & 7.2-3.9 / 13.3-7.2 & 5 \\
\hline $101 / S$ & Anneleen Knutsen & $\mathrm{T}$ & 187 & $6.7 / 3.4$ & 4.9-1.0 / 9.1-1.9 & 1 \\
\hline $102 / S$ & Carten Elina & CS & 102 & $10.2 / 5.2$ & 6.3-1.7 / 11.7-3.1 & 4 \\
\hline $103 / \mathrm{S}$ & Corinne & CS & 74 & $9.5 / 4.9$ & 5.8-1.0 / 10.7-1.9 & 1 \\
\hline $104 / \mathrm{S}$ & Edmy & CS & 86 & $9.9 / 5.1$ & $8.6-3.7 / 15.9-6.9$ & 2 \\
\hline $105 / S$ & Pluto & $\mathrm{T}$ & 184 & 10.2 / 5.2 & 7.4-2.7 / 13.7-5.0 & 3 \\
\hline $106 / S$ & Samskip Frost & CS & 86 & 13.2 / 6.8 & $5.2-1.1 / 9.6-2.0$ & 2 \\
\hline 107/S & Seapike & $\mathrm{T}$ & 200 & 12.8 / 6.6 & 5.9-0.6 / 10.9-1.1 & 3 \\
\hline $108 / \mathrm{S}$ & Stavangerfjord & $\mathrm{P}$ & 170 & $14.5 / 7.5$ & 8.7-2.6 / 16.1-4.8 & 4 \\
\hline \multicolumn{7}{|c|}{ Crossing courses } \\
\hline $109 / \mathrm{V}$ & Bulk Switzerland & B & 289 & $9.5 / 4.9$ & $20.0-18.0$ / 37.0-33.3 & 5 \\
\hline $110 / \mathrm{V}$ & Free Neptune & CS & 185 & 11.5 / 5.9 & $14.2-11.5$ / 26.2-21.3 & 2 \\
\hline $111 / \mathrm{V}$ & MSC Rachele & C & 334 & $19.5 / 10.0$ & 23.4-17.5 / 43.3-32.4 & 5 \\
\hline $112 / \mathrm{V}$ & NCC Danah & $\mathrm{T}$ & 183 & $13.5 / 6.9$ & 5.6-3.3 / 10.4-6.1 & 7 \\
\hline $113 / \mathrm{V}$ & OOCL Korea & $\mathrm{C}$ & 366 & 15.8 / 8.1 & 5.7-1.6 / 10.6-3.0 & $7 \mathrm{sw}$ \\
\hline $114 / \mathrm{V}$ & Spirit of Britain & $\mathrm{F}$ & 213 & $23.5 / 12.1$ & $9.1-8.3$ / 16.9-22.4 & 3 \\
\hline 115/L & Cap San Marco & $\mathrm{C}$ & 333 & $20.0 / 10.3$ & 4.3-2.7 / 8.0-5.0 & 4 \\
\hline $116 / \mathrm{L}$ & Carnival Valor & $\mathrm{P}$ & 292 & 18.2 / 9.4 & $3.4-2.9$ / 6.3-5.4 & 4 \\
\hline 117/L & Horncap & $\mathrm{C}$ & 153 & 14.5 / 7.5 & 5.6-2.9 / 10.4-5.4 & 3 \\
\hline 118/L & JS Columbia & B & 199 & $14.4 / 7.4$ & 16.7-12.8 / 30.9-23.7 & 2 \\
\hline 119/L & Gpo Grace & CS & 225 & $2.4 / 1.2$ & 9.1-5.5 / 16.9-10.2 & $4 \mathrm{sw}$ \\
\hline 120/L & Rix Spring & D & 82 & $2.3 / 1.2$ & 3.9-2.4 / 7.2-4.4 & $3 \mathrm{sw}$ \\
\hline 121/M & Abis Calais & CS & 115 & $9.4 / 4.8$ & $16-13.7$ / 29.6-25.4 & 4 \\
\hline 122/M & Arklow Cadet & CS & 87 & 10.6 / 5.4 & $6.7-4.3 / 12.4-8.0$ & 3 \\
\hline 123/M & Coral Lophelia & $\mathrm{T}$ & 109 & $13.4 / 6.9$ & 17.1-16.3 / 31.7-30.2 & 5 \\
\hline 124/M & Ilyas Efendiyev & CS & 140 & $8.4 / 4.3$ & 9.6-4.9 / 17.8-9.1 & 2 \\
\hline 125/M & Rio de Janeiro Express & CS & 260 & $13.4 / 7.4$ & 19.5-15.4 / 36.1-28.5 & 2 \\
\hline 126/M & Sea Faith & $\mathrm{T}$ & 182 & $12.8 / 6.6$ & 10.9-7.7 / 20.2-14.3 & 3 \\
\hline 127/M & Leon Poseidon & $\mathrm{T}$ & 149 & $13.5 / 6.9$ & $16.0-12.5$ / 29.6-23.2 & 3 \\
\hline $128 / \mathrm{M}$ & Lolland & $\mathrm{F}$ & 100 & $12.3 / 6.3$ & $2.8-0.9 / 5.2-1.7$ & 8 \\
\hline 129/M & Thun Gazelle & $\mathrm{T}$ & 116 & $9.9 / 5.1$ & $6.7-5.4 / 12.4-10.0$ & 5 \\
\hline $130 / \mathrm{M}$ & Washington Express & $\mathrm{C}$ & 243 & 14.2 / 7.3 & $12.2-7.8$ / 22.6-14.4 & 3 \\
\hline 131/M & Sakura & B & 196 & $11.2 / 5.8$ & 10.9-10.1 / 20.2-18.7 & 3 \\
\hline 132/M & Vienna Wood N & B & 190 & 10.6 / 5.4 & $8.1-6.2$ / 15.0-11.5 & 3 \\
\hline 133/M & Arklow Vanguard & CS & 87 & $11.3 / 5.8$ & 6.9-3.1 / 12.8-5.7 & 2 \\
\hline 134/M & Seastar Trojan & CS & 179 & $12.3 / 6.3$ & $15.9-14.3$ / 29.4-26.5 & 6 \\
\hline 135/M & Med Baltic & $\mathrm{T}$ & 123 & $13.6 / 7.0$ & $5.8-4.6 / 10.7-8.5$ & 1 \\
\hline 136/M & Nave Equinox & $\mathrm{T}$ & 184 & $13.5 / 6.9$ & 10.6-7.5 / 19.6-13.9 & 1 \\
\hline 137/M & Pac Athena & CS & 179 & 13.8 / 7.1 & 10.5-6.0 / 19.5-11.1 & 2 \\
\hline 138/M & Melody Fair & CS & 200 & $12.9 / 6.6$ & $15.9-12.8$ / 29.4-23.7 & 1 \\
\hline 139/M & Nissos Schinoussa & $\mathrm{T}$ & 254 & $7.9 / 4.1$ & 9.5-7.5 / 17.6-13.9 & 1 \\
\hline
\end{tabular}




\begin{tabular}{|c|c|c|c|c|c|c|}
\hline 140/M & Bomar Victory & C & 179 & $7.2 / 3.7$ & $21.8-20.5 / 40.4-38.0$ & 2 \\
\hline $141 / \mathrm{M}$ & Ocean Tianchen & B & 199 & $2.2 / 1.1$ & 6.1-5.1 / 11.3-9.4 & 7 \\
\hline $142 / \mathrm{M}$ & Apl Le Havre & $\mathrm{C}$ & 354 & $16.4 / 8.4$ & $21.2-19.5$ / 39.3-36.1 & 6 \\
\hline $143 / \mathrm{M}$ & Salahuddi N & $\mathrm{C}$ & 368 & 17.2 / 8.8 & $15.3-14.7$ / 28.3-27.2 & 6 \\
\hline 144/M & Bulk Beothuk & B & 190 & $10.7 / 5.5$ & 18.7-15.7 34.6-29.1 & 6 \\
\hline $145 / \mathrm{M}$ & Atlantic Geneva & $\mathrm{C}$ & 148 & $10.8 / 5.6$ & 24.3-18.3 / 45.0-33.9 & 5 \\
\hline 146/M & Green Ocean & CS & 108 & $11.3 / 5.8$ & $18.9-15.6$ / 35.0-28.9 & 5 \\
\hline $147 / \mathrm{M}$ & Am Gijon & $\mathrm{B}$ & 292 & $10.6 / 5.5$ & 20.0-19.1 / 37.0-35.4 & 5 \\
\hline $148 / \mathrm{M}$ & Hestia Leader & $\mathrm{RO}$ & 199 & $16.5 / 8.5$ & $16.8-15.1$ / 31.1-28.0 & 2 \\
\hline $149 / \mathrm{M}$ & Volcan de Tijarafe & $\mathrm{P}$ & 154 & $23.6 / 12.1$ & $11.7-9.1 / 21.7-16.9$ & 2 \\
\hline $150 / \mathrm{M}$ & Brotonne Bridge & $\mathrm{C}$ & 268 & $13.5 / 6.9$ & 7.7-0.9 / 14.3-1.7 & 4 \\
\hline $151 / \mathrm{M}$ & Genoa Express & $\mathrm{C}$ & 228 & $11.1 / 5.7$ & $10.3-9.7$ / 19.1-18.0 & 5 \\
\hline $152 / S$ & Ekfjord & $\mathrm{T}$ & 144 & 11.7 / 6.0 & $8.6-2.4$ / 15.9-4.4 & 3 \\
\hline $153 / S$ & Freya & CS & 118 & 17.5 / 9.0 & $8.8-5.8 / 16.3-10.7$ & 1 \\
\hline $154 / S$ & John August Essberger & $\mathrm{T}$ & 120 & 12.2 / 6.3 & $10.3-5.5$ / 19.1-10.2 & 1 \\
\hline $155 / S$ & King Gregory & $\mathrm{T}$ & 183 & 11.7 / 6.0 & $8.6-3.1 / 15.9-5.7$ & 3 \\
\hline $156 / S$ & Jade & CS & 190 & 12.6 / 6.5 & $5.2-1.5 / 9.6-2.8$ & $3 \mathrm{sw}$ \\
\hline $157 / S$ & Navion Stavanger & CS & 277 & 13.5 / 6.9 & $11.9-6.1 / 22.0-11.3$ & 5 \\
\hline $158 / S$ & Ionis & $\mathrm{P}$ & 96 & $15.6 / 8.0$ & $2.9-2.3 / 5.4-4.3$ & 3 \\
\hline $159 / S$ & Tamara & CS & 117 & $12.4 / 6.4$ & $16.6-15.9$ / 30.7-29.4 & 2 \\
\hline $160 / S$ & Floto SG 244 & FV & 18 & $2.8 / 1.4$ & $2.9-0.5 / 5.4-0.9$ & 3 \\
\hline $161 / S$ & Superspeed 1 & $\mathrm{P}$ & 26 & $25.5 / 13.1$ & $4.0-1.8 / 7.4-3.3$ & 3 \\
\hline \multicolumn{7}{|c|}{ Both ships at anchor } \\
\hline $162 / \mathrm{M}$ & Despina Angel & CS & 169 & 0 & $1.2 / 2.2$ & 3 \\
\hline $163 / \mathrm{M}$ & Lacerta & $\mathrm{T}$ & 183 & 0 & $1.7 / 3.2$ & 3 \\
\hline $164 / \mathrm{M}$ & Ridgebury Maryselena & $\mathrm{T}$ & 274 & 0 & $1.0 / 1.8$ & 3 \\
\hline $165 / \mathrm{M}$ & Dylan & $\mathrm{T}$ & 180 & 0 & $1.9 / 3.5$ & 3 \\
\hline
\end{tabular}

\section{RESULTS OF THE MEASUREMENTS}

Tables 4 and 5 present results of tests listed in Table 3 and conducted on ships mentioned in the Table 2 . They contain information about mean values $(\mathrm{M})$ and standard deviations $(\sigma)$ of true motion vectors (true courses and true speeds) and CPA of observed vessels presented by AIS and ARPA in particular measurement series. Values of standard deviations exceeding their limits specified in Table 1 are printed in these tables in bold and are underlined.

Table 4. Results of tests conducted on ships. True course and true speed (95\% probability figures) [own study]

\begin{tabular}{|c|c|c|c|c|c|c|c|c|}
\hline \multirow{4}{*}{$\begin{array}{l}\text { ize } \\
\text { category }\end{array}$} & \multirow{2}{*}{\multicolumn{2}{|c|}{ ARPA }} & \multicolumn{6}{|c|}{ AIS } \\
\hline & & & \multicolumn{2}{|c|}{ True speed (TSp) } & \multicolumn{2}{|c|}{ True course (TC) } & \multicolumn{2}{|c|}{ True speed (TSp) } \\
\hline & M & $2 \sigma$ & M & $2 \sigma$ & M & $2 \sigma$ & M & $2 \sigma$ \\
\hline & {$\left[{ }^{\circ}\right]$} & {$\left[{ }^{\circ}\right]$} & {$[\mathrm{kn} / \mathrm{m} / \mathrm{s}]$} & {$[\mathrm{kn} / \mathrm{m} / \mathrm{s}]$} & {$\left[{ }^{\circ}\right]$} & {$\left[{ }^{\circ}\right]$} & {$[\mathrm{kn} / \mathrm{m} / \mathrm{s}]$} & {$[\mathrm{kn} / \mathrm{m} / \mathrm{s}]$} \\
\hline \multicolumn{9}{|c|}{ Parallel courses - overtaking } \\
\hline $1 / \mathrm{V}$ & 255.0 & 2.7 & $13.12 / 6.74$ & $0,28 / 0.14$ & 254.5 & 3.0 & $12.96 / 6.66$ & $0.12 / 0.06$ \\
\hline $2 / \mathrm{V}$ & 252.6 & 142.7 & $0.43 / 0.22$ & $1.30 / 0.67$ & 265.3 & 67.8 & 0.00 & 0.00 \\
\hline $3 / \mathrm{V}$ & 202.5 & 196.4 & 0.00 & 0.00 & 353.7 & 3.2 & $0.06 / 0.03$ & $0.10 / 0.05$ \\
\hline $4 / \mathrm{V}$ & 026.7 & $\underline{15.9}$ & 11.36./ 5.84 & $2.32 / 1.19$ & 026.0 & 9.6 & 11.14 / 5.73 & $0.28 / 0.14$ \\
\hline $5 / \mathrm{V}$ & 248.7 & $\overline{3.3}$ & $8.34 / 4.29$ & $\overline{0.16 / 0,08}$ & 246.0 & $\overline{4.1}$ & $8.06 / 4.14$ & $0.34 / 0.17$ \\
\hline $6 / \mathrm{V}$ & 264.5 & 23.0 & $11.06 / 5.68$ & $0.96 / 0.49$ & 264.1 & 12.3 & $10.86 / 5.58$ & $0.44 / 0.23$ \\
\hline $7 / \mathrm{L}$ & 094.3 & $\overline{12.7}$ & $11.96 / 6.15$ & $\overline{1.20 / 0.62}$ & 092.9 & $\overline{4.1}$ & $11.96 / 6.15$ & $0.10 / 0.05$ \\
\hline $8 / \mathrm{L}$ & 086.0 & 1.9 & $13.38 / 6.88$ & $\overline{0.66 / 0.34}$ & 085.4 & 1.4 & $13.31 / 6.84$ & $0.08 / 0.04$ \\
\hline 9/L & 084.4 & 1.5 & $14.98 / 7.70$ & $\overline{0.22 / 0.11}$ & 082.5 & 1.3 & $14.05 / 7.22$ & $0.10 / 0.05$ \\
\hline 10/L & 160.7 & 1.9 & $11.65 / 5.99$ & $0.26 / 0.13$ & 161.7 & $\underline{8.8}$ & $11.8 / 6.07$ & $0.44 / 0.23$ \\
\hline $11 / \mathrm{L}$ & 187.1 & $\underline{12.9}$ & $11.48 / 5.90$ & $\underline{6.20 / 3.12}$ & 188.3 & $\underline{5.1}$ & $11.51 / 5.92$ & $1.50 / 0.77$ \\
\hline $12 / \mathrm{L}$ & 023.5 & $\overline{68.7}$ & $19.01 / 9.77$ & $\overline{11.12 / 5.72}$ & 039.0 & $\overline{0.3}$ & $15.46 / 7.95$ & $\overline{0.10 / 0.05}$ \\
\hline 13/M & 029.6 & $\overline{1.0}$ & $17.44 / 8.96$ & $0,30 / 0.15$ & 029.4 & 1.0 & $17.48 / 8.98$ & $0.34 / 0.17$ \\
\hline $14 / \mathrm{M}$ & 206.9 & $\underline{8.4}$ & 9.17 / 4,71 & $0,28 / 0.14$ & 208.6 & 4.2 & $9.08 / 4.67$ & $0.16 / 0.08$ \\
\hline 15/M & 051.4 & $\overline{2.4}$ & $15.07 / 7.75$ & $0,18 / 0.09$ & 051.1 & 4.5 & $15.09 / 7.76$ & $0.28 / 0.14$ \\
\hline $16 / \mathrm{M}$ & 061.1 & 0.5 & $11.10 / 5.71$ & $0,12 / 0.06$ & 060.9 & 0.9 & $11.10 / 5.71$ & $0.10 / 0.05$ \\
\hline $17 / \mathrm{M}$ & 189.3 & 23.3 & $0.50 / 0.26$ & $0,16 / 0.08$ & 149.7 & 13.3 & $0.52 / 0.27$ & $0.10 / 0.05$ \\
\hline $18 / \mathrm{M}$ & 035.2 & 4.3 & $18.22 / 9.37$ & $0,54 / 0.28$ & 034.7 & 2.9 & $18.19 / 9.35$ & $0.16 / 0.08$ \\
\hline 19/M & 211.2 & 2.0 & $10.73 / 5.52$ & $\overline{0,38 / 0.20}$ & 210.9 & 1.1 & $10.69 / 5.49$ & $0.22 / 0.11$ \\
\hline $20 / \mathrm{M}$ & 347.1 & 3.8 & $12.06 / 6.20$ & $1.44 / 0.74$ & 346.9 & 3.3 & $11.87 / 6.10$ & $0.42 / 0.22$ \\
\hline $21 / \mathrm{M}$ & 194.6 & 4.6 & $10.69 / 5.49$ & $\overline{0.68 / 0.35}$ & 194.2 & 4.5 & $10.86 / 5.58$ & $0.46 / 0.24$ \\
\hline $22 / \mathrm{M}$ & 242.6 & 2.8 & $10.24 / 5.26$ & $\overline{0.30 / 0.15}$ & 242.7 & 1.1 & $10.25 / 5.27$ & $0.12 / 0.06$ \\
\hline $23 / \mathrm{M}$ & 250.8 & 3.4 & $4.72 / 2.43$ & $0.30 / 0.15$ & 249.2 & 1.9 & $4.78 / 2.46$ & $0.08 / 0.04$ \\
\hline $24 / \mathrm{M}$ & 179.3 & 2.7 & $16.18 / 8.32$ & $0.42 / 0.22$ & 179.4 & 1.9 & $16.13 / 8.29$ & $0.22 / 0.11$ \\
\hline $25 / \mathrm{M}$ & 140.7 & 4.0 & $11.79 / 6.06$ & $\underline{0.80 / 0.41}$ & 139.2 & 1.8 & $11.65 / 5.99$ & $0.20 / 0.10$ \\
\hline $26 / \mathrm{M}$ & 252.9 & 1.3 & $11.30 / 5.81$ & $\overline{0.58 / 0.30}$ & 253.6 & 1.2 & $11.21 / 5.76$ & $0.08 / 0.04$ \\
\hline $27 / \mathrm{M}$ & 244.8 & 1.8 & $17.96 / 9.23$ & $\overline{1.34 / 0.69}$ & 247.3 & 0.9 & $18.00 / 9.25$ & $0.10 / 0.05$ \\
\hline $28 / \mathrm{M}$ & 072.2 & 0.6 & $14.51 / 7.46$ & $\overline{0.64 / 0.33}$ & 072.5 & 1.1 & $14.57 / 7.49$ & $0.16 / 0.08$ \\
\hline 29/M & 236.5 & 1.0 & $14.15 / 27.53$ & $\overline{0.38 / 0.20}$ & 236.9 & 0.7 & $14.23 / 7.31$ & $0.16 / 0.08$ \\
\hline $30 / \mathrm{M}$ & 062.6 & $\underline{229.9}$ & $0.49 / 0.25$ & $0.48 / 0.25$ & 060.2 & $\underline{8.1}$ & $0.48 / 0.25$ & $\underline{0.65 / 0.33}$ \\
\hline $31 / \mathrm{M}$ & 247.3 & 3.1 & 5.65 / 2.90 & $\underline{0.59 / 0.30}$ & 248.7 & $\underline{6.2}$ & 5.66 / 2.91 & $\underline{0.62 / 0.32}$ \\
\hline $32 / \mathrm{M}$ & 248.9 & 1.2 & $11.88 / 6.11$ & $0.31 / 0.16$ & 248.9 & 2.0 & $11.81 / 6.07$ & $0.15 / 0.08$ \\
\hline
\end{tabular}




\begin{tabular}{|c|c|c|c|c|c|c|c|c|}
\hline $33 / \mathrm{M}$ & 251.6 & 2.0 & $6.18 / 3.18$ & $\underline{0.54 / 0.28}$ & 251.3 & $\underline{5.3}$ & $6.19 / 3.18$ & $0.40 / 0.21$ \\
\hline $34 / \mathrm{M}$ & 253.7 & 3.4 & 13.60 / 6.99 & $0.41 / 0.21$ & 252.7 & 2.8 & 13.50 / 6.94 & $0.21 / 0.11$ \\
\hline $35 / \mathrm{M}$ & 210.7 & 1.7 & $11.25 / 5.78$ & $0.39 / 0.20$ & 210.6 & 3.1 & $11.21 / 5.76$ & $0.22 / 0.11$ \\
\hline $36 / \mathrm{M}$ & 097.6 & 4.1 & $2.81 / 1.44$ & $0.15 / 0.08$ & 094.5 & $\underline{7.6}$ & 2.89 / 1.49 & $0.20 / 0.10$ \\
\hline $37 / \mathrm{M}$ & 024.4 & 4.0 & 9.09 / 4.68 & 0.47 / 0.24 & 033.0 & 1.0 & $8.95 / 4.60$ & $0.10 / 0.05$ \\
\hline 38/M & 111.9 & $\underline{12.8}$ & $17.71 / 9.10$ & $2.62 / 1.35$ & 108.6 & 1.6 & 16.96 / 8.72 & $0.10 / 0.05$ \\
\hline 39/M & 256.6 & $\underline{10.1}$ & 4.83 / 2.48 & $1.18 / 0.61$ & 254.4 & $\underline{14.3}$ & 4.79 / 2.46 & $\underline{0.95 / 0.49}$ \\
\hline $40 / \mathrm{M}$ & 076.5 & 3.1 & $21.21 / 10.90$ & $\underline{7.80 / 4.01}$ & 078.7 & 1.3 & 18.24 / 9.38 & $0.26 / 0.13$ \\
\hline $41 / \mathrm{M}$ & 262.6 & $\underline{6.5}$ & 16.79 / 8.63 & $\underline{1.61 / 0.83}$ & 263.9 & 2.9 & 16.72 / 8.59 & $0.27 / 0.14$ \\
\hline $42 / \mathrm{S}$ & 037.1 & 3.1 & $17.00 / 8.74$ & $0.18 / 0.09$ & 037.5 & 2.0 & 17.00 / 8.74 & $0.16 / 0.08$ \\
\hline $43 / S$ & 249.8 & 0.6 & $22.80 / 11.7$ & $0.36 / 0.19$ & 249.6 & 1.1 & $22.70 / 11.67$ & $0.16 / 0.08$ \\
\hline $44 / S$ & 034.3 & 1.8 & $13.0 / 6.68$ & $0.16 / 0.08$ & 033.9 & 1.8 & 13.00 / 6.68 & $0.12 / 0.06$ \\
\hline $45 / S$ & 051.1 & 0.8 & 18.5 / 9.51 & 0.34 / 0.17 & 051.1 & 1.0 & $18.40 / 9.46$ & 0.34 / 0.17 \\
\hline $46 / S$ & 231.5 & 0.3 & $9.71 / 4.99$ & 1.2 / 0.62 & 231.6 & 0.9 & $9.60 / 4.93$ & 0.14 / 0.07 \\
\hline $47 / S$ & 034.8 & 1.8 & $11.50 / 5.91$ & $1.5 / 0.78$ & 034.8 & 2.0 & 11.50 / 5.91 & $0.18 / 0.09$ \\
\hline $48 / \mathrm{S}$ & 061.0 & 2.4 & $11.60 / 5.96$ & $0.40 / 0.21$ & 060.9 & 1.8 & 12.00 / 6.17 & $0.18 / 0.09$ \\
\hline $49 / \mathrm{S}$ & 085.7 & 4.1 & 4.33 / 2.23 & $\underline{0.64 / 0.33}$ & 085.5 & 2.0 & 4.38 / 2.25 & 0.09 / 0.05 \\
\hline $50 / \mathrm{S}$ & 359.4 & 1.3 & $12.20 / 6.27$ & $0.26 / 0.13$ & 000.1 & 1.0 & 12.17 / 6.26 & $0.14 / 0.07$ \\
\hline $51 / \mathrm{S}$ & 029.0 & 2.8 & $10.61 / 5.45$ & $\underline{0.60 / 0.31}$ & 028.7 & 5.0 & $10.46 / 5.38$ & $0.45 / 0.23$ \\
\hline $52 / S$ & 289.6 & 1.1 & $10.00 / 5.14$ & $0.28 / 0.15$ & 289.6 & 1.3 & 10.00 / 5.14 & $0.12 / 0.06$ \\
\hline $53 / S$ & 250.5 & 0.7 & $10.65 / 5.47$ & $0.41 / 0.21$ & 250.8 & 1.7 & 10.49 / 5.39 & $0.38 / 0.20$ \\
\hline $54 / \mathrm{S}$ & 208.0 & 0.8 & $18.38 / 9.45$ & $\underline{0.76 / 0.39}$ & 207.7 & 1.4 & 18.44 / 9.48 & $0.21 / 0.11$ \\
\hline $55 / \mathrm{S}$ & Variable & - & $0.88 / 0.45$ & $\underline{0.86 / 0.44}$ & Variable & - & $0.54 / 0.28$ & 0.42 / 0.22 \\
\hline $56 / S$ & 102.6 & 2.7 & $5.70 / 2.93$ & $\underline{1.37 / 0.70}$ & 103.3 & 3.0 & $5.66 / 2.91$ & $1.70 / 0.87$ \\
\hline $57 / S$ & 083.3 & 1.3 & $9.20 / 4.73$ & $0.20 / 0.10$ & 082.9 & 1.4 & 9.19 / 4.72 & 0.12 / 0.06 \\
\hline $58 / \mathrm{S}$ & 159.3 & $\underline{13.1}$ & $5.03 / 2.59$ & $\underline{3.40 / 1.75}$ & 160.1 & 3.7 & $4.97 / 2.55$ & $0.15 / 0.08$ \\
\hline $59 / \mathrm{S}$ & 166.9 & $\underline{18.2}$ & $9.11 / 4.68$ & $\underline{0.63 / 0.32}$ & 166.9 & $\underline{18.0}$ & $9.03 / 4.64$ & $0.29 / 0.15$ \\
\hline $60 / S$ & 155.4 & $\underline{147.0}$ & $0.53 / 0.27$ & $0.45 / 0.23$ & 120.6 & $\underline{125.7}$ & 0.17 / 0.09 & $0.18 / 0.09$ \\
\hline $61 / S$ & 158.7 & 3.5 & $7.80 / 4.01$ & $0.28 / 0.14$ & 158.9 & 2.7 & $7.78 / 4.00$ & $0.23 / 0.12$ \\
\hline \multicolumn{9}{|c|}{ Reciprocal courses } \\
\hline $62 / \mathrm{V}$ & 068.4 & $\underline{7.1}$ & $19.26 / 9.90$ & $2.98 / 1.53$ & 068.7 & 1.4 & 19.17 / 9.85 & $0.22 / 0.11$ \\
\hline $63 / \mathrm{V}$ & 207.8 & 1.2 & $14.03 / 7.21$ & $0.28 / 0.14$ & 204.9 & 1.1 & 13.99 / 7.19 & 0.14 / 0.07 \\
\hline $64 / \mathrm{L}$ & 232.2 & $\underline{5.3}$ & $11.16 / 5,74$ & $0.22 / 0.11$ & 232.7 & 2.4 & 11.14 / 5.73 & 0.12 / 0.06 \\
\hline $65 / \mathrm{L}$ & 040.7 & 1.3 & $11.73 / 6.03$ & $0.38 / 0.20$ & 042.6 & 1.2 & 11.52 / 5.92 & $0.16 / 0.08$ \\
\hline 66/L & 236.6 & 1.2 & 15.54 / 7.99 & $0.50 / 0.26$ & 237.1 & 1.0 & 15.43 / 7.93 & $0.18 / 0,09$ \\
\hline $67 / \mathrm{L}$ & 054.2 & 3.1 & 14.62 / 7.51 & $\underline{0.72 / 0.37}$ & 052.1 & 1.0 & 14.11 / 7.25 & $0.08 / 0.04$ \\
\hline 68/L & 229.6 & 1.5 & 10.39 / 5.34 & $0.24 / 0.12$ & 234.5 & 1.8 & 10.18 / 5.23 & 0.12 / 0.06 \\
\hline 69/L & 321.3 & 1.4 & 13.10 / 6.73 & $0.30 / 0.15$ & 322.5 & 2.2 & 13.07 / 6.72 & 0.44 / 0.23 \\
\hline 70/L & 163.0 & $\underline{17.7}$ & $11.91 / 6.12$ & $\underline{1.18 / 0.61}$ & 160.3 & $\underline{10.2}$ & 11.12 / 5.72 & $0.28 / 0.14$ \\
\hline 71/L & 139.6 & 2.1 & $12.38 / 6.36$ & $0.48 / 0.25$ & 139.5 & 2.1 & 12.42 / 6.38 & $0.13 / 0.07$ \\
\hline $72 / \mathrm{M}$ & 232.9 & 2.4 & $11.28 / 5.80$ & $\underline{0.64 / 0.33}$ & 231.4 & 1.6 & 11.20 / 5.76 & $0.16 / 0.08$ \\
\hline 73/M & 009.2 & 4.4 & $13.06 / 6.71$ & $1.00 / 0.51$ & 011.1 & 1.3 & 12.78 / 6.57 & $0.26 / 0.13$ \\
\hline $74 / \mathrm{M}$ & 312.5 & $\underline{6.1}$ & $15.57 / 8.00$ & $\underline{0.58 / 0.30}$ & 314.6 & 1.9 & 15.43 / 7.93 & $0.16 / 0.08$ \\
\hline 75/M & 221.1 & 0.9 & $12.57 / 6.46$ & $1.50 / 0.77$ & 220.4 & 1.1 & 12.12 / 6.23 & $0.16 / 0.08$ \\
\hline $76 / \mathrm{M}$ & 207.2 & 2.5 & $9.31 / 4.79$ & $\underline{0.54 / 0.28}$ & 209.1 & 1.3 & $9.30 / 4.78$ & $0.16 / 0.08$ \\
\hline 77/M & 180.9 & $\underline{7.4}$ & 9.33 / 4.80 & $0.46 / 0.24$ & 181.2 & 1.5 & $9.23 / 4.74$ & $0.08 / 0.04$ \\
\hline 78/M & 179.2 & 4.1 & 14.47 / 7.44 & $0.48 / 0.25$ & 181.6 & 1.6 & 14.34 / 7.37 & $0.16 / 0.08$ \\
\hline 79/M & 178.9 & 2.8 & 14.39 / 7.40 & $0.26 / 0.13$ & 181.7 & 1.4 & 14.25 / 7.32 & 0.12 / 0.06 \\
\hline $80 / \mathrm{M}$ & 032.2 & $\underline{7.8}$ & 12.39 / 6.37 & $0.22 / 0.11$ & 032.0 & 1.2 & 12.39 / 6.37 & $0.10 / 0.05$ \\
\hline $81 / \mathrm{M}$ & 326.8 & 3.8 & 11.86 / 6.10 & $\underline{0.65 / 0.33}$ & 329.1 & 2.5 & 11.26 / 5.79 & 0.17 / 0.09 \\
\hline $82 / \mathrm{M}$ & 091.2 & 3.3 & 13.47 / 6.92 & $\underline{0.85 / 0.44}$ & 091.9 & 0.8 & 13.20 / 6.78 & 0 \\
\hline 83/M & 208.7 & 1.7 & 16.60 / 8.53 & $\underline{0.56 / 0.29}$ & 210.1 & 1.6 & $16.41 / 8.43$ & $0.25 / 0.13$ \\
\hline $84 / \mathrm{M}$ & 208.8 & 2.2 & 13.42 / 6.90 & $0.45 / 0.23$ & 209.3 & 1.0 & 13.30 / 6.84 & $0.10 / 0.05$ \\
\hline $85 / \mathrm{M}$ & 026.0 & 1.4 & 12.25 / 6.30 & 0.49 / 0.25 & 027.9 & 3.1 & 12.10 / 6.22 & $0.16 / 0.08$ \\
\hline $86 / \mathrm{M}$ & 021.1 & 1.4 & 12.54 / 6.45 & $\underline{0.54 / 0.28}$ & 023.3 & 3.1 & 12.40 / 6.38 & $0.21 / 0.11$ \\
\hline $87 / \mathrm{M}$ & 030.5 & 0.7 & 10.87 / 5.59 & 0.17 / 0.32 & 031.7 & 0.9 & 10.80 / 5.55 & $0.13 / 0.24$ \\
\hline $88 / \mathrm{M}$ & 071.4 & 1.7 & 20.48 / 10.53 & 0.35 / 0.18 & 069.7 & 2.6 & 20.37 / 10.47 & $0.46 / 0.24$ \\
\hline $89 / \mathrm{M}$ & 037.1 & 1.9 & $13.58 / 6.98$ & $\underline{0.66 / 0.34}$ & 043.3 & 1.8 & 13.54 / 6.96 & $0.21 / 0.11$ \\
\hline 90/M & 104.4 & 1.2 & 9.83 / 5.05 & $0.13 / 0.07$ & 109.6 & 1.8 & 9.84 / 5.06 & $0.16 / 0.08$ \\
\hline 91/M & 078.5 & 1.0 & $10.40 / 5.35$ & $\underline{1.2 / 0.62}$ & 077.9 & 4.8 & 10.0 / 5.14 & $0.14 / 0.07$ \\
\hline $92 / \mathrm{S}$ & 058.7 & 1.6 & $11.30 / 5.81$ & $\underline{1.14 / 0.59}$ & 058.3 & 0.9 & 11.11 / 5.71 & $\underline{0.80 / 0.41}$ \\
\hline $93 / \mathrm{S}$ & 070.9 & 0.7 & $10.30 / 5.29$ & $0.28 / 0.14$ & 070.2 & 0.4 & 10.20 / 5.24 & $0.1 / 0.05$ \\
\hline $94 / S$ & 037.5 & 1.2 & $17.05 / 8.76$ & $\underline{0.56 / 0.29}$ & 037.3 & 0.7 & 16.90 / 8.69 & $0.24 / 0.12$ \\
\hline $95 / \mathrm{S}$ & 268.1 & 0.8 & $10.58 / 5.44$ & $0.28 / 0.14$ & 268.8 & 0.6 & 10.45 / 5.37 & $0.10 / 0.05$ \\
\hline $96 / \mathrm{S}$ & 099.2 & 2.2 & $17.00 / 8.74$ & $\underline{0.83 / 0.43}$ & 098.9 & 1.7 & 16.86 / 8.67 & $\underline{0.63 / 0.32}$ \\
\hline $97 / S$ & 214.7 & 2.5 & $14.17 / 7.28$ & $\underline{0.57 / 0.29}$ & 216.4 & 1.7 & 14.20 / 7.30 & $0.05 / 0.03$ \\
\hline $98 / S$ & 017.9 & 1.4 & $12.97 / 6.67$ & $0.48 / 0.25$ & 016.3 & 3.6 & 13.14 / 6.75 & 0.24 / 0.12 \\
\hline $99 / \mathrm{S}$ & 284.3 & 2.0 & $12.05 / 6.19$ & $\underline{0.75 / 0.39}$ & 285.6 & 2.0 & 12.20 / 6.27 & 0.39 / 0.20 \\
\hline $100 / S$ & 033.7 & 2.5 & $12.21 / 6.28$ & $\underline{1.12 / 0.58}$ & 035.8 & 0.5 & 12.30 / 6.32 & 0 \\
\hline $101 / S$ & 270.7 & 3.5 & $7.06 / 3.63$ & $\underline{1.28 / 0.66}$ & 269.5 & 1.3 & 6.72 / 3.45 & $0.10 / 0.05$ \\
\hline $102 / S$ & 045.4 & 4.3 & $11.0 / 5.65$ & $\underline{0.74 / 0.38}$ & 043.3 & 1.7 & $10.21 / 5.25$ & $3.43 / 1.76$ \\
\hline $103 / S$ & 226.3 & $\underline{5.5}$ & $9.60 / 4.93$ & $0.35 / 0.18$ & 224.8 & 1.3 & $9.51 / 4.89$ & $0.18 / 0.09$ \\
\hline $104 / S$ & 053.2 & $\underline{6.0}$ & $9.88 / 5.08$ & $0.30 / 0.15$ & 050.8 & 1.1 & 9.87 / 5.07 & 0.14 / 0.07 \\
\hline $105 / S$ & 041.6 & 4.4 & $10.38 / 5.34$ & 0.22 / 0.11 & 034.5 & 1.0 & 10.18 / 5.23 & 0.12 / 0.06 \\
\hline $106 / S$ & 285.5 & 4.7 & 13.27 / 6.82 & 0.35 / 0.18 & 284.8 & 1.5 & 13.18 / 6.77 & $0.27 / 0.14$ \\
\hline
\end{tabular}




\begin{tabular}{|c|c|c|c|c|c|c|c|c|}
\hline $107 / S$ & 148.8 & 4.1 & $12.80 / 6.58$ & $\underline{0.82 / 0.42}$ & 147.1 & 2.4 & 12.77 / 6.56 & $0.26 / 0.13$ \\
\hline $108 / S$ & 132.6 & 3.6 & $14.40 / 7.40$ & $\overline{0.60 / 0.31}$ & 130.7 & 3.3 & $14.46 / 7.43$ & $0.30 / 0.15$ \\
\hline \multicolumn{9}{|c|}{ Crossing courses } \\
\hline $109 / \mathrm{V}$ & 084.6 & $\underline{12.5}$ & $9.62 / 4.94$ & $3.94 / 2.03$ & 084.2 & 8.7 & $9.53 / 4.90$ & $0.22 / 0.11$ \\
\hline $110 / \mathrm{V}$ & 025.9 & 11.4 & $11.49 / 5.91$ & $0.56 / 0.29$ & 024.8 & 1.2 & $11.48 / 5.90$ & $0.20 / 0.10$ \\
\hline $111 / \mathrm{V}$ & 289.4 & 16.4 & $20.11 / 10.34$ & $7.82 / 4.02$ & 289.7 & 1.4 & 19.72 / 10.14 & $0.14 / 0.07$ \\
\hline $112 / \mathrm{V}$ & 087.5 & 3.1 & $13.69 / 7.04$ & $0.38 / 0.20$ & 087.8 & 7.6 & $13.56 / 6.97$ & $0.40 / 0.21$ \\
\hline $113 / \mathrm{V}$ & 270.5 & 1.3 & 15.97 / 8.21 & $0.60 / 0.31$ & 270.5 & 2.2 & $15.96 / 8.20$ & $0.38 / 0.20$ \\
\hline $114 / \mathrm{V}$ & 118.4 & 5.8 & $23.14 / 11.89$ & $0.90 / 0.46$ & 114.7 & 7.3 & $23.50 / 12.01$ & $0.48 / 0.25$ \\
\hline $115 / \mathrm{L}$ & 198.8 & 0.5 & $20.43 / 10.50$ & $0.36 / 0.19$ & 202.0 & 0.5 & 20.05 / 10.31 & $0.14 / 0.07$ \\
\hline $116 / \mathrm{L}$ & 130.0 & 1.3 & $18.38 / 9.45$ & $\underline{0.52 / 0.27}$ & 130.0 & 1.1 & $18.16 / 9.33$ & $0.12 / 0.06$ \\
\hline 117/L & 017.8 & 1.1 & $14.32 / 7.36$ & $\overline{0.32 / 0.16}$ & 019.6 & 1.6 & $14.60 / 7.50$ & $0.12 / 0.06$ \\
\hline $118 / \mathrm{L}$ & 033.1 & 2.5 & 13.84 / 7.11 & $0.70 / 0.36$ & 034.4 & 3.1 & $14.29 / 7.35$ & $0.16 / 0.08$ \\
\hline $119 / \mathrm{L}$ & 158.8 & 16.2 & $2.56 / 1.32$ & $0.72 / 0.37$ & 161.2 & 6.6 & $2.44 / 1.25$ & $0.52 / 0.27$ \\
\hline $120 / \mathrm{L}$ & 030.9 & 16.9 & $9.58 / 4.92$ & $2.11 / 1.08$ & 028.3 & $\frac{2.1}{2.1}$ & $9.48 / 4.87$ & $0.30 / 0.15$ \\
\hline $121 / \mathrm{M}$ & 166.9 & 1.9 & $9.55 / 4.91$ & $0.92 / 0.47$ & 165.9 & 2.5 & $9.40 / 4.83$ & $0.22 / 0.11$ \\
\hline $122 / \mathrm{M}$ & 298.0 & 2.5 & $10.62 / 5.46$ & $0.28 / 0.14$ & 297.4 & 3.8 & 10.49 / 5.39 & $0.18 / 0.09$ \\
\hline $123 / \mathrm{M}$ & 215.4 & 9.4 & 13.12 / 6.74 & $\underline{2.40 / 1.23}$ & 216.9 & 1.9 & 13.45 / 6.91 & $0.20 / 0.10$ \\
\hline $124 / \mathrm{M}$ & 269.0 & $\underline{5.8}$ & $8.55 / 4.39$ & $0.84 / 0.43$ & 268.8 & 2.0 & 8.44 / 4.34 & $0.12 / 0.06$ \\
\hline $125 / \mathrm{M}$ & 083.5 & 2.3 & $14.56 / 7.48$ & $0.70 / 0.36$ & 083.6 & 0.8 & $14.46 / 7.43$ & $0.10 / 0.05$ \\
\hline $126 / \mathrm{M}$ & 310.6 & 0.6 & 12.83 / 6.59 & $0.42 / 0.22$ & 311.2 & 1.4 & $12.80 / 6.58$ & $0.17 / 0.09$ \\
\hline $127 / \mathrm{M}$ & 324.9 & 3.5 & 13.55 / 6.96 & $0.59 / 0.30$ & 324.6 & 3.8 & 13.52 / 6.95 & $0.07 / 0.04$ \\
\hline $128 / \mathrm{M}$ & 126.7 & 6.2 & 12.27 / 6.31 & $1.17 / 0.60$ & 125.1 & 3.9 & 11.84 / 6.09 & $0.21 / 0.11$ \\
\hline $129 / \mathrm{M}$ & 349.7 & $\overline{1.3}$ & $9.86 / 5.09$ & $0.14 / 0.07$ & 351.3 & 1.3 & $9.74 / 5.01$ & $0.20 / 0.10$ \\
\hline $130 / \mathrm{M}$ & 290.4 & 2.0 & $14.16 / 7.29$ & $0.76 / 0.39$ & 290.9 & 1.3 & $14.43 / 7.42$ & $0.34 / 0.17$ \\
\hline 131/M & 116.4 & 1.5 & $11.20 / 6.27$ & $0.37 / 0.19$ & 116.1 & 1.5 & $11.21 / 5.76$ & $0.08 / 0.04$ \\
\hline 132/M & 107.8 & 1.6 & $10.56 / 5.43$ & $0.31 / 0.16$ & 108.6 & 0.9 & $10.78 / 5.54$ & $0.07 / 0.04$ \\
\hline $133 / \mathrm{M}$ & 200.5 & 1.9 & $11.32 / 5.82$ & 0.44 / 023 & 202.4 & 0.9 & $10.96 / 5.63$ & $0.11 / 0.06$ \\
\hline $134 / \mathrm{M}$ & 116.6 & 6.3 & $12.63 / 6.49$ & $2.08 / 1.10$ & 117.6 & 1.7 & 12.27 / 6.31 & $0.40 / 0.21$ \\
\hline $135 / \mathrm{M}$ & 005.7 & 6.3 & $14.03 / 7.21$ & $0.66 / 0.34$ & 009.6 & 6.5 & 13.57 / 6.97 & $0.09 / 0.05$ \\
\hline $136 / \mathrm{M}$ & 074.3 & 9.1 & 13.60 / 6.99 & $0.65 / 0.33$ & 075.1 & $\overline{1.0}$ & 13.48 / 6.93 & $0.18 / 0.09$ \\
\hline $137 / \mathrm{M}$ & 266.2 & 2.3 & 13.52 / 6.95 & $0.83 / 0.43$ & 270.3 & 1.8 & 13.77 / 7.08 & $0.10 / 0.05$ \\
\hline $138 / \mathrm{M}$ & 253.1 & 9.6 & 11.95 / 6.14 & $3.04 / 1.56$ & 263.1 & 1.0 & 12.92 / 6.64 & $0.56 / 0.29$ \\
\hline $139 / \mathrm{M}$ & 290.3 & 4.2 & $6.63 / 3.41$ & $2.08 / 1.07$ & 294.0 & 1.3 & $7.87 / 4.05$ & $0.76 / 0.39$ \\
\hline 140/M & 129.5 & 2,0 & $7.36 / 3.78$ & $\overline{0.10 / 0.05}$ & 123.7 & 2.6 & $7.15 / 3.68$ & $\overline{0.13 / 0.07}$ \\
\hline $141 / \mathrm{M}$ & 201.4 & 89.52 & $2.44 / 1.25$ & $1.79 / 0.92$ & 210.9 & 51.9 & $2.23 / 1.15$ & $1.67 / 0.86$ \\
\hline $142 / \mathrm{M}$ & 265.8 & 38.8 & 17.49 / 8.99 & $11.66 / 5.99$ & 257.8 & 34.3 & 16.37 / 8.41 & $10.16 / 5.22$ \\
\hline $143 / \mathrm{M}$ & 076.1 & $\underline{17.1}$ & $17.63 / 9.06$ & $8.51 / 4.37$ & 080.4 & 4.0 & 17.18 / 8.83 & $\underline{0.64 / 0.33}$ \\
\hline $144 / \mathrm{M}$ & 088.9 & $\overline{54.1}$ & $10.47 / 5.38$ & $\overline{5.91 / 3.04}$ & 079.8 & 9.1 & $10.69 / 5.49$ & $\overline{0.42 / 0.22}$ \\
\hline $145 / \mathrm{M}$ & 059.7 & $\overline{122.3}$ & 13.40 / 6.89 & $\overline{10.50 / 5.40}$ & 033.8 & $\overline{9.62}$ & $10.83 / 5.57$ & $1.69 / 0.87$ \\
\hline $146 / \mathrm{M}$ & 086.2 & 34.7 & 12.16 / 6.25 & $14.47 / 7.44$ & 085.4 & $\underline{5.5}$ & 11.32 / 5.82 & $0.33 / 0.17$ \\
\hline 147/M & 093.7 & $\overline{101.2}$ & 10.59 / 5.44 & $\overline{16.23 / 8.34}$ & 081.7 & $\overline{9.4}$ & $10.55 / 5.42$ & $0.40 / 0.21$ \\
\hline $148 / \mathrm{M}$ & 208.9 & 5 & 16.33 / 8.39 & $2.98 / 1.53$ & 210.4 & 4.0 & 16.47 / 8.47 & $0.13 / 0.07$ \\
\hline $149 / \mathrm{M}$ & 097.0 & 3.1 & $23.72 / 12.19$ & $1.00 / 0.51$ & 099.2 & 2.7 & $23.62 / 12.14$ & $0.29 / 0.15$ \\
\hline $150 / \mathrm{M}$ & 252.6 & 3.3 & $13.35 / 6.86$ & $\overline{0.29 / 0.15}$ & 256.0 & 3.2 & 13.47 / 6.92 & $0.13 / 0.07$ \\
\hline $151 / \mathrm{M}$ & 105.6 & 4.8 & $11.56 / 5.94$ & $\underline{0.56 / 0.29}$ & 107.1 & 1.4 & 11.14 / 5.73 & $0.15 / 0.08$ \\
\hline $152 / S$ & 237.7 & 1.0 & 11.86 / 6.10 & $0.22 / 0.11$ & 238.5 & 1.1 & 11.62 / 5.97 & $0.08 / 0.04$ \\
\hline $153 / S$ & 017.3 & 2.4 & $17.60 / 9.05$ & $0.60 / 0.31$ & 017.7 & 0.1 & 17.50 / 9.00 & $0.16 / 0.08$ \\
\hline $154 / S$ & 015.8 & 3.2 & $12.20 / 6.27$ & $0.44 / 0.23$ & 016.6 & 0.9 & 12.17 / 6.27 & $0.18 / 0.09$ \\
\hline $155 / S$ & 254.2 & 2.4 & 11.90 / 6.12 & $1.58 / 0.81$ & 255.2 & 0.8 & 11.70 / 6.01 & $0.28 / 0.14$ \\
\hline $156 / S$ & 066.5 & 8.5 & 12.84 / 6.60 & $0.53 / 0.27$ & 064.9 & 6.5 & 12.69 / 6.52 & $0.19 / 0.10$ \\
\hline $157 / S$ & 252.2 & $\overline{2.4}$ & $13.58 / 6.98$ & $0.73 / 0.38$ & 251.0 & 2.8 & 13.70 / 7.04 & $0.41 / 0.21$ \\
\hline $158 / S$ & 050.6 & 140.8 & $16.03 / 8.24$ & $\overline{12.15 / 6.25}$ & 032.3 & 10.7 & $15.60 / 8.02$ & $0.13 / 0.07$ \\
\hline $159 / S$ & 313.7 & 15.6 & 12.39 / 6.37 & $1.73 / 0.89$ & 314.5 & 1.5 & $12.38 / 6.36$ & $0.13 / 0.07$ \\
\hline $160 / S$ & 205.9 & 2.3 & $2.91 / 1.50$ & $1.03 / 0.53$ & 206.4 & $\underline{6.7}$ & $2.79 / 1.43$ & $0.08 / 0.04$ \\
\hline $161 / S$ & 183.5 & 1.3 & 25.12 / 12.91 & $0.60 / 0.31$ & 182.3 & 0.5 & $25.5 / 13.11$ & $0.21 / 0.11$ \\
\hline \multicolumn{9}{|c|}{ Both ships at anchor } \\
\hline $162 / \mathrm{M}$ & $1.21 / 2.24$ & $01 / 0.02$ & $0.12 / 0.06$ & $0.12 / 0.06$ & $1.23 / 2.28$ & $0.01 / 0.02$ & 0 & 0 \\
\hline $163 / \mathrm{M}$ & $1.72 / 3.19$ & $0.02 / 0.04$ & $0.25 / 0.13$ & $0.35 / 0.18$ & $1.69 / 3.13$ & $0.07 / 0.13$ & $0.06 / 0.04$ & 0.09 / 0.05 \\
\hline $164 / \mathrm{M}$ & $0.99 / 1.83$ & $0.02 / 0.04$ & $0.28 / 0.14$ & $0.21 / 0.11$ & $1.00 / 1.85$ & $0.05 / 0.09$ & $0.43 / 0.22$ & $0.45 / 0.23$ \\
\hline $165 / \mathrm{M}$ & $1.87 / 3.46$ & $0.01 / 0.02$ & $0.27 / 0.14$ & $0.33 / 0.17$ & $1.83 / 3.39$ & $0.01 / 0.02$ & 0 & 0 \\
\hline
\end{tabular}

Table 5. Results of tests conducted on ship's. CPA (95\% probability figures) [own study]

\begin{tabular}{lcccc}
\hline $\begin{array}{l}\text { No/s } \\
\text { ize } \\
\text { category }\end{array}$ & $\begin{array}{c}\text { CPA (ARPA) } \\
\text { Mean value } \\
{[\mathrm{M} / \mathrm{km}]}\end{array}$ & $\begin{array}{c}2 \sigma \\
{[\mathrm{M} / \mathrm{km}]}\end{array}$ & $\begin{array}{c}\text { CPA (AIS) } \\
\text { Mean value } \\
{[\mathrm{M} / \mathrm{km}]}\end{array}$ & $\begin{array}{c}2 \sigma \\
{[\mathrm{M} / \mathrm{km}]}\end{array}$ \\
\hline \multicolumn{2}{r}{ Parallel courses - overtaking } & & & \\
\hline 1/V & $0.69 / 1.28$ & $0.04 / 0.07$ & $0.67 / 1.24$ & $0.04 / 0.07$ \\
2/V & $4.50 / 8.33$ & $\underline{\mathbf{1 . 7 4} / \mathbf{3 . 2 2}}$ & $4.38 / 8.11$ & $\underline{\mathbf{0 . 7 4} / \mathbf{1 . 3 7}}$ \\
$3 / \mathrm{V}$ & $0.68 / 1.26$ & $0.02 / 0.04$ & $0.69 / 1.28$ & $0.04 / 0.07$ \\
4/V & $17.18 / 31.82$ & $\underline{\mathbf{5 . 2 0} / \mathbf{9 . 6 3}}$ & $15.71 / 29.09$ & $\underline{\mathbf{6 . 1 2} / \mathbf{1 1 . 3 3}}$ \\
$5 / \mathrm{V}$ & $2.65 / 4.91$ & $0.14 / 0.26$ & $2.58 / 4.78$ & $\underline{\mathbf{7 . 0 4} / \mathbf{1 3 . 0 4}}$ \\
6/V & $11.31 / 20.95$ & $\underline{\mathbf{8 . 6 8 / 1 6 . 0 8}}$ & $9.99 / 18.50$ & $\underline{\mathbf{1 . 6 0} / \mathbf{2 . 9 6}}$ \\
$7 / \mathrm{L}$ & $6.72 / 12.45$ & $\underline{\mathbf{5 . 8 2 / 1 0 . 7 8}}$ & $7.66 / 14.19$ & $0.06 / 0.11$
\end{tabular}




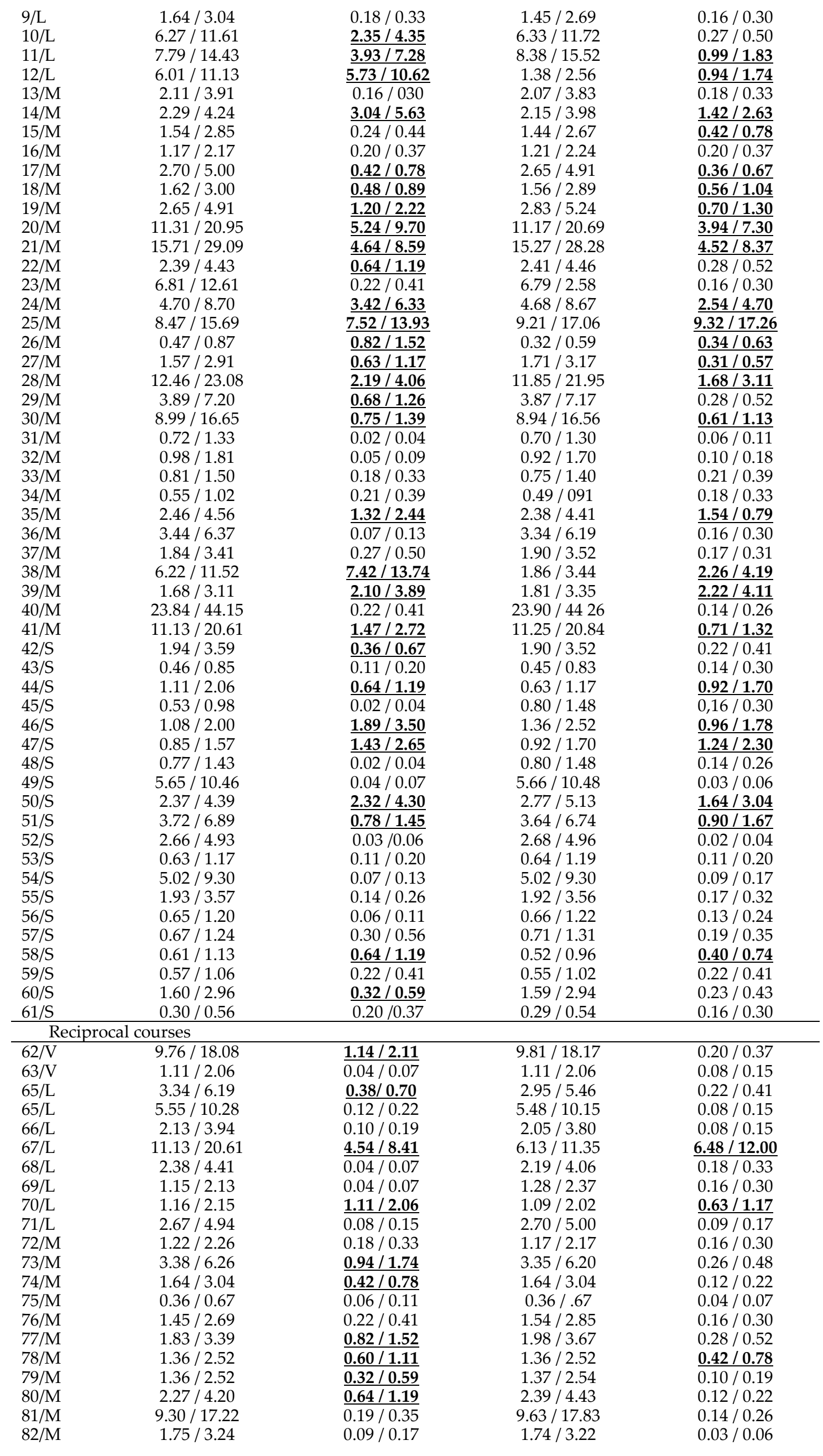




\begin{tabular}{|c|c|c|c|c|}
\hline & & & & \\
\hline $83 / \mathrm{M}$ & $0.66 / 1.22$ & $0.11 / 0.20$ & $0.66 / 1.22$ & $0.10 / 0.19$ \\
\hline $84 / \mathrm{M}$ & $0.70 / 1.30$ & $0.12 / 0.22$ & $0.69 / 1.28$ & $0.08 / 0.15$ \\
\hline $85 / \mathrm{M}$ & $4.66 / 8.63$ & $0.05 / 0.09$ & $4.61 / 8.54$ & $0.10 / 0.19$ \\
\hline $86 / \mathrm{M}$ & $4.36 / 8.08$ & $0.04 / 0.07$ & $4.31 / 7.98$ & $0.06 / 0.11$ \\
\hline $87 / \mathrm{M}$ & 0.73 / 1.35 & $0.06 / 0.11$ & 0.74 / 1.37 & $0.08 / 0.15$ \\
\hline $88 / \mathrm{M}$ & $1.24 / 2.30$ & $0.20 / 0.37$ & $1.24 / 2.30$ & $0.30 / 0.56$ \\
\hline 89/M & $3.66 / 6.78$ & $0.12 / 0.22$ & $3.66 / 6.78$ & $0.11 / 0.20$ \\
\hline $90 / \mathrm{M}$ & $1.80 / 3.33$ & $0.17 / 0.31$ & $1.91 / 3.54$ & $0.14 / 0.26$ \\
\hline 91/M & 7.38 / 13.67 & $0.18 / 0.33$ & $7.39 / 13.69$ & $0.10 / 0.19$ \\
\hline $92 / S$ & 1.37 / 2.54 & 0.19 / 0.36 & $1.2 / 2.22$ & $0.06 / 0.11$ \\
\hline $93 / S$ & $3.50 / 6.48$ & $0.04 / 0.07$ & $3.50 / 6.48$ & $0.08 / 0.15$ \\
\hline $94 / S$ & $5.02 / 9.30$ & $0.08 / 0.15$ & $5.01 / 9.28$ & $0.24 / 0.44$ \\
\hline $95 / S$ & $1.81 / 3.35$ & $\underline{0.94 / 1.74}$ & 1.69 / 3.13 & $0.01 / 0.02$ \\
\hline $96 / S$ & $1.42 / 2.63$ & $\overline{0.19 / 0.35}$ & $1.41 / 2.61$ & $0.16 / 0.30$ \\
\hline $97 / S$ & $5.26 / 9.74$ & $0.13 / 0.24$ & $5.62 / 10.41$ & $0.11 / 0.20$ \\
\hline $98 / S$ & 4.69 / 8.69 & $0.13 / 0.24$ & 4.69 / 8.69 & $0.14 / 0.26$ \\
\hline $99 / \mathrm{S}$ & 4.52 / 8.37 & $0.10 / 0.19$ & $4.56 / 8.45$ & $0.11 / 0.20$ \\
\hline $100 / S$ & $3.86 / 7.15$ & $0.12 / 0.22$ & 3.88 / 7.19 & $0.08 / 0.15$ \\
\hline $101 / S$ & $0.67 / 1.24$ & $0.17 / 0.31$ & $0.71 / 1.31$ & $0.09 / 0.17$ \\
\hline $102 / S$ & $0.56 / 1.04$ & $0.24 / 0.44$ & 0.53 / 0.98 & 0.14 / 0.26 \\
\hline $103 / S$ & $0.50 / 0.93$ & $0.26 / 0.48$ & 0.49 / 0.91 & $0.05 / 0.09$ \\
\hline $104 / S$ & 1.02 / 1.89 & $\underline{0.38 / 0.70}$ & $1.07 / 1.98$ & $0.06 / 0.11$ \\
\hline $105 / S$ & $1.01 / 1.87$ & $\overline{0.28 / 0.52}$ & $0.91 / 1.69$ & $0.13 / 0.24$ \\
\hline $106 / S$ & $0.53 / 0.98$ & $0.25 / 0.46$ & $0.55 / 1.11$ & $0.05 / 0.09$ \\
\hline $107 / S$ & $0.61 / 1.13$ & $0.12 / 0.22$ & $0.64 / 1.19$ & $0.07 / 0.13$ \\
\hline $108 / S$ & $1.08 / 2.00$ & $0.26 / 0.48$ & $1.07 / 1.98$ & $0.27 / 0.50$ \\
\hline \multicolumn{5}{|c|}{ Crossing courses } \\
\hline $109 / \mathrm{V}$ & $17.18 / 31.82$ & $\underline{0.76 / 1.41}$ & 17.19 / 31.84 & $\underline{0.50 / 0.93}$ \\
\hline 110/V & 9.43 / 17.46 & $\underline{1.22 / 2.26}$ & 9.48 / 17.56 & $0.16 / 0.30$ \\
\hline $111 / \mathrm{V}$ & $3.85 / 7.13$ & $4.64 / 8.59$ & $3.65 / 6.76$ & $0.30 / 0.56$ \\
\hline $112 / \mathrm{V}$ & $2.97 / 5.50$ & $\overline{0.26 / 0.48}$ & $2.92 / 5.41$ & $0.22 / 0.41$ \\
\hline $113 / \mathrm{V}$ & $1.51 / 2.80$ & $0.12 / 0.22$ & $1.47 / 2.72$ & $0.16 / 0.30$ \\
\hline $114 / \mathrm{V}$ & $8.28 / 15.33$ & $0.06 / 0.11$ & $8.25 / 15.28$ & $0.06 / 0.11$ \\
\hline 115/L & $2.60 / 4.82$ & $0.04 / 0.07$ & $2.54 / 4.70$ & $0.10 / 01.9$ \\
\hline 116/L & $2.90 / 5.37$ & $0.02 / 0.04$ & 2.79 / 5.17 & $0.04 / 0.07$ \\
\hline 117/L & 2.84 / 5.26 & $0.04 / 0.07$ & $2.70 / 5.00$ & 0.12 / 0.22 \\
\hline $118 / \mathrm{L}$ & 5.92 / 10.96 & $\underline{0.52 / 0.96}$ & $5.30 / 9.82$ & $\underline{0.32 / 0.59}$ \\
\hline 119/L & $2.05 / 3.80$ & $0.29 / 0.54$ & $2.06 / 3.82$ & $\underline{0.34 / 0.63}$ \\
\hline $120 / \mathrm{L}$ & $2.17 / 4.02$ & $\underline{1.03 / 1.91}$ & $2.27 / 4.20$ & $\overline{0.33 / 0.61}$ \\
\hline $121 \mathrm{M}$ & $4.43 / 8.20$ & $\underline{1.86 / 3.44}$ & $4.33 / 8.02$ & $\underline{0.42 / 0.78}$ \\
\hline $122 / \mathrm{M}$ & 3.72 / 6.89 & $\overline{0.14 / 0.26}$ & $3.71 / 6.87$ & $\overline{014 / 0.26}$ \\
\hline $123 / \mathrm{M}$ & 13.12 / 24.30 & 6.44 / 11.93 & 14.30 / 26.48 & $\underline{0.32 / 0.59}$ \\
\hline $124 / \mathrm{M}$ & 2.44 / 4.52 & $\underline{0.58 / 1.07}$ & $2.41 / 4.46$ & $0.20 / 0.37$ \\
\hline $125 / \mathrm{M}$ & $0.76 / 1.41$ & $\overline{0.66 / 1.22}$ & $0.77 / 1.43$ & $\underline{0.32 / 0.59}$ \\
\hline $126 / \mathrm{M}$ & $3.73 / 6,91$ & $\underline{0.32 / 0.59}$ & $3.72 / 6.89$ & $\overline{0.22 / 0.41}$ \\
\hline $127 / \mathrm{M}$ & 7.89 / 14.61 & $\overline{0.25 / 0.46}$ & $8.00 / 14.82$ & $\underline{0.40 / 0.74}$ \\
\hline $128 / \mathrm{M}$ & $0.98 / 1.81$ & $0.07 / 0.13$ & $0.92 / 1.70$ & $\overline{0.03 / 0.06}$ \\
\hline $129 / \mathrm{M}$ & $4.11 / 7.61$ & $0.18 / 0.33$ & 3.94 / 7.30 & $0.22 / 0.41$ \\
\hline 130/M & $5.23 / 9.69$ & $0.29 / 0.54$ & 5.32 / 9.85 & 0.14 / 0.26 \\
\hline $131 / \mathrm{M}$ & 9.26 / 17.15 & $0.22 / 0.41$ & $9.21 / 17.06$ & $0.11 / 020$ \\
\hline 132/M & 3.29 / 6.09 & $\underline{0.34 / 0} \quad 0.63$ & 3.08 / 5.70 & $0.10 / 0.19$ \\
\hline $133 / \mathrm{M}$ & 2.59 / 4.80 & $\overline{0.09 / 0.17}$ & $2.53 / 4.69$ & $0.03 / 0.06$ \\
\hline $134 / \mathrm{M}$ & 13.04 / 24.2 & $\underline{1,05 / 1.94}$ & 12.90 / 23.89 & $\underline{0.88 / 1.63}$ \\
\hline $135 / \mathrm{M}$ & $3.36 / 6.22$ & $\overline{0.10 / 0.19}$ & 3.37 / 6.24 & $\overline{0.09 / 0.17}$ \\
\hline $136 / \mathrm{M}$ & 5.84 / 10.82 & $\underline{0.52 / 0.96}$ & 5.89 / 10.91 & $0.16 / 0.30$ \\
\hline $137 / \mathrm{M}$ & 5.85 / 10.83 & $\overline{0.25 / 0.46}$ & $5.81 / 10.76$ & $0.21 / 0.39$ \\
\hline $138 / \mathrm{M}$ & $15.58 / 28.85$ & $0.14 / 0.26$ & 15.60 / 28.89 & $0.04 / 0.07$ \\
\hline $139 / \mathrm{M}$ & 7.42 / 13.74 & $0.17 / 0.31$ & $7.28 / 13.48$ & $0.17 / 0.31$ \\
\hline $140 / \mathrm{M}$ & $1.87 / 3.46$ & $\underline{2.65 / 4.91}$ & $0.91 / 1.69$ & 1.27 / 2.35 \\
\hline $141 / \mathrm{M}$ & $4.41 / 8.17$ & $\overline{1.60 / 2.96}$ & . & - \\
\hline $142 / \mathrm{M}$ & 12.73 / 23.58 & $\overline{9.57 / 17.72}$ & 14.32 / 26.52 & $\underline{9.85 / 18.24}$ \\
\hline $143 / \mathrm{M}$ & 14.88 / 27.56 & $\overline{0.27 / 0.50}$ & 14.88 / 27.56 & $\underline{0.38 / 0.70}$ \\
\hline $144 / \mathrm{M}$ & 13.52 / 25.04 & $\underline{5.76 / 10.67}$ & 14.48 / 26.82 & $1.19 / 2.20$ \\
\hline $145 / \mathrm{M}$ & 7.15 / 13.24 & $\overline{10.3219 .11}$ & 2.67 / 4.94 & $2.23 / 4.13$ \\
\hline $146 / \mathrm{M}$ & 15.95 / 29.54 & $2.08 / 3.85$ & $16.24 / 30.08$ & $\underline{0.57 / 1.06}$ \\
\hline $147 / \mathrm{M}$ & $18.50 / 34.26$ & $3.33 / 6.17$ & $19.06 / 35.30$ & $\overline{0.43 / 0.80}$ \\
\hline $148 / \mathrm{M}$ & 15.14 / 28.04 & $\overline{0.25 / 0.46}$ & 15.12 / 28.00 & $\overline{0.17 / 0.31}$ \\
\hline $149 / \mathrm{M}$ & 9.08 / 16.82 & $0.11 / 0.20$ & 9.13 / 16.91 & $0.16 / 0.30$ \\
\hline $150 / \mathrm{M}$ & $0.83 / 1.54$ & $0.23 / 0.43$ & 0.84 / 1.56 & $0.15 / 0.28$ \\
\hline $151 / \mathrm{M}$ & 9.69 / 17.95 & $\underline{0.33 / 0.61}$ & 9.69 / 17.95 & $0.20 / 0.37$ \\
\hline $152 / S$ & $2.39 / 4.43$ & $0.04 / 0.07$ & 2.37 / 4.39 & $0.10 / 0.19$ \\
\hline $153 / S$ & $5.38 / 9.96$ & $\underline{0.32 / 0.59}$ & $5.67 / 10.50$ & $0.18 / 0.33$ \\
\hline $154 / S$ & $5.50 / 10.19$ & $0.12 / 0.22$ & $5.41 / 10.02$ & $0.10 / 0.18$ \\
\hline $155 / S$ & $3.20 / 5.93$ & $0.04 / 0.07$ & $3.13 / 5.80$ & $0.16 / 0.30$ \\
\hline $156 / S$ & 1.36 / 2.52 & $\underline{0.34 / 0.63}$ & $1.36 / 2.52$ & $0.26 / 0.48$ \\
\hline
\end{tabular}




\begin{tabular}{lcccc}
$157 / S$ & $2.47 / 4.57$ & $0.29 / 0.54$ & $2.47 / 4.57$ & $0.30 / 0.56$ \\
$158 / S$ & $1.63 / 3.02$ & $\underline{\mathbf{0 . 9 9 / 1 . 8 3}}$ & $1.70 / 3.15$ & $0.20 / 0.37$ \\
$159 / S$ & $14.28 / 26.5$ & $\underline{\mathbf{3 . 3 3} / \mathbf{6 . 1 7}}$ & $14.63 / 27.10$ & $\underline{\mathbf{0 . 4 1} / 0.76}$ \\
$160 / \mathrm{S}$ & $0.46 / 0.85$ & $0.13 / 0.24$ & $0.45 / 0.83$ & $0.07 / 0.13$ \\
$161 / \mathrm{S}$ & $1.74 / 3.22$ & $0.12 / 0.22$ & $1.74 / 3.22$ & $0.02 / 0.03$ \\
\hline \multicolumn{2}{l}{ Both ships at anchor } & & & \\
\hline $162 / \mathrm{M}$ & $0.55 / 1.02$ & $\underline{\mathbf{0 . 5 8 / 1 . 0 7}}$ & $0.92 / 1.70$ & $\underline{\mathbf{0 . 5 6 / 1 . 0 4}}$ \\
$163 / \mathrm{M}$ & $1.19 / 2.20$ & $\underline{\mathbf{0 . 6 6 / 1 . 7 8}}$ & $0.39 / 0.72$ & $\underline{\mathbf{0 . 5 6} / \mathbf{1 . 0 4}}$ \\
$164 / \mathrm{M}$ & $0.60 / 1.11$ & $\underline{\mathbf{0 . 6 2} / \mathbf{1 . 1 5}}$ & $0.95 / 1.80$ & $0.26 / 0.48$ \\
$165 / \mathrm{M}$ & $1.51 / 2.80$ & $\mathbf{0 . 8 9 / \mathbf { 1 . 6 5 }}$ & $1.37 / 2.54$ & $\mathbf{0 . 4 4 / 0 . 8 1}$ \\
\hline
\end{tabular}

\section{DISCUSSION OF THE TESTS RESULTS}

Table 6 summarizes the information presented in Tables 4 and 5 and shows the number of particular types of investigated meeting situations of two ships where errors (for $95 \%$ probability) of the presented data were greater than their values specified in the international standards and shown in Table 1.

Table 6. The number of meeting situations where errors of data presented by AIS and ARPA (CPA, true course and / or true speed) were greater than their values presented in Table 1 (for $95 \%$ probability figures, excluding both ships at anchor) [own study]

\begin{tabular}{lllllllll}
\hline Data & \multicolumn{6}{l}{$\begin{array}{l}\text { Number of meeting situations with data } \\
\text { errors greater than their values presented in } \\
\end{array}$} \\
& $\begin{array}{l}\text { Table 1 } \\
\text { ARPA }\end{array}$ & AIS & & & & \\
& 1 & 2 & 3 & All & 1 & 2 & 3 & All \\
TC & 1 & 1 & 0 & 2 & 6 & 0 & 6 & 12 \\
TSp & 7 & 19 & 12 & 38 & 1 & 3 & 2 & 6 \\
TC \& TSp & 2 & 0 & 6 & 8 & 1 & 0 & 0 & 1 \\
CPA & 9 & 3 & 3 & 15 & 23 & 2 & 9 & 34 \\
CPA \& TC & 4 & 4 & 0 & 8 & 4 & 1 & 4 & 9 \\
CPA \& TSp & 10 & 2 & 5 & 17 & 0 & 0 & 0 & 0 \\
All data & 10 & 3 & 17 & 30 & 3 & 0 & 5 & 8 \\
\hline$\Sigma / \Sigma_{\mathrm{T}}$ & $43 /$ & $32 /$ & $43 /$ & $118 /$ & $38 /$ & $6 /$ & $26 /$ & $70 /$ \\
& 61 & 47 & 53 & 161 & 61 & 47 & 53 & 161 \\
\hline
\end{tabular}

Abbreviations used in Table 6 mean:

1 - parallel courses - overtaking;

2 - reciprocal courses;

3 - crossing courses;

All - all meeting situations;

TC - true course;

TSp - true speed; and

$\Sigma / \Sigma_{\mathrm{T}}$ - number of meeting situations of a given type with data errors greater than presented in Table 1 / total number of all meeting situations of this type.

Table 7 presents correlation between the errors of the results of ARPA calculation and errors of the true course, true speed and/or CPA indications by AIS for the same ships' meeting situations.

The analysis of the data contained in Tables 4 and 5 shows that the differences between the average values of the true course $(\triangle T C)$, true speed $(\triangle T S p)$ and/or CPA $(\triangle \mathrm{CPA})$ indicated by the AIS and ARPA for particular observed and tracked vessels exceeded errors defined in the IMO Resolution MSC.192(79) and IEC Standard 61993-2 in 40 measurement series ( $24 \%$ of all of them). It should be noted that they exceeded these errors in $29.5 \%$ of measurements for ships overtaking on parallel courses, $24.5 \%$ for ships at crossing courses, and 19\% for vessels sailing on reciprocal courses. Detailed information on these meeting situations is presented in Table 8.

Table 7. Correlation between errors of the results of ARPA calculation and errors of the true course, true speed and/or CPA indications by AIS for the same meeting situation (excluding both ships at anchor, abbreviations as in Table 7) [own study]

\begin{tabular}{|c|c|c|c|c|c|c|c|c|c|c|}
\hline \multirow{2}{*}{$\begin{array}{l}\text { Data presented by } \\
\text { ARPA with errors } \\
\text { greater than } \\
\text { presented in Table } 1\end{array}$} & \multicolumn{2}{|c|}{$\begin{array}{l}\text { Meeting } \\
\text { situation }\end{array}$} & \multicolumn{8}{|c|}{$\begin{array}{l}\text { The number of meeting situations in which AIS simultaneously presented } \\
\text { particular data with errors greater than presented in Table } 1\end{array}$} \\
\hline & Type & No & TC & TSp & $\begin{array}{l}\text { TC \& } \\
\text { TSp }\end{array}$ & CPA & $\begin{array}{l}\text { CPA \& } \\
\text { TC }\end{array}$ & $\begin{array}{l}\text { CPA \& } \\
\text { TSp }\end{array}$ & All data & $\mathrm{OK}$ \\
\hline \multirow{5}{*}{$\mathrm{TC}$} & 1 & 1 & - & - & - & - & - & - & - & 1 \\
\hline & 2 & 1 & - & - & - & - & - & - & - & 1 \\
\hline & 3 & - & - & - & - & - & - & - & - & - \\
\hline & $\Sigma$ & 2 & - & - & - & - & - & - & - & 2 \\
\hline & 1 & 7 & 1 & 1 & 1 & - & - & - & - & 4 \\
\hline \multirow{3}{*}{ TSp } & 2 & 19 & - & 3 & - & - & - & - & - & 16 \\
\hline & 3 & 12 & 1 & 1 & - & 1 & - & - & - & 9 \\
\hline & $\Sigma$ & 38 & 2 & 5 & 1 & 1 & - & - & - & 29 \\
\hline \multirow{4}{*}{ TC \& TSp } & 1 & 2 & 2 & - & - & - & - & - & - & - \\
\hline & 2 & - & - & - & - & - & - & - & - & - \\
\hline & 3 & 6 & 2 & 1 & - & - & - & - & 2 & 1 \\
\hline & $\Sigma$ & 8 & 4 & 1 & - & - & - & - & 2 & 1 \\
\hline \multirow{4}{*}{ CPA } & 1 & 9 & 1 & - & - & 6 & - & - & - & 2 \\
\hline & 2 & 3 & - & - & - & 1 & - & - & - & 2 \\
\hline & 3 & 3 & - & - & - & 1 & - & - & - & 2 \\
\hline & $\Sigma$ & 15 & 1 & - & - & 8 & - & - & - & 6 \\
\hline \multirow{5}{*}{ CPA \& TC } & 1 & 4 & 1 & - & - & 1 & 1 & - & 1 & - \\
\hline & 2 & 4 & - & - & - & - & - & - & - & 4 \\
\hline & 3 & - & - & - & - & - & - & - & - & - \\
\hline & $\Sigma$ & 8 & 1 & - & - & 1 & 1 & - & 1 & 4 \\
\hline & 1 & 10 & - & - & - & 10 & - & - & - & - \\
\hline \multirow{3}{*}{ CPA \& TSp } & 2 & 2 & - & - & - & 1 & - & - & - & 1 \\
\hline & 3 & 5 & - & - & - & 3 & - & - & - & 2 \\
\hline & $\Sigma$ & 17 & - & - & - & 14 & - & - & - & 3 \\
\hline All data & 1 & 10 & - & - & - & 5 & 3 & - & 2 & - \\
\hline
\end{tabular}




\begin{tabular}{ccccccccccc} 
& 2 & 3 & - & - & - & - & 1 & - & - & 2 \\
& 3 & 17 & 2 & - & - & 4 & 4 & - & 3 & 4 \\
& $\Sigma$ & 30 & 2 & - & - & 9 & 8 & - & 5 & 6 \\
\multirow{4}{*}{ OK } & 1 & 18 & 1 & - & - & 1 & - & - & - & 16 \\
& 2 & 15 & - & - & - & - & - & - & - & 15 \\
& 3 & 10 & 1 & - & - & - & - & - & - & 9 \\
& $\Sigma$ & 43 & 2 & - & - & 1 & - & - & - & 40 \\
\end{tabular}

\footnotetext{
Abbreviations used in Table 7 mean:

Type 1, 23 - see Table 6;

OK - data presented by AIS without unacceptable errors;

TC - true course;

TSp - true speed; and

$\Sigma$ - all meeting situations.
}

Table 8. Meeting situations in which the differences in the indications of average values of true course, true speed and CPA by ARPA and AIS are less than the error of determining these parameters presented in the IMO Resolution MSC.192(79) and IEC Standard 61993-2 [own study]

\begin{tabular}{|c|c|c|c|c|c|c|c|c|}
\hline No & Noms & $\Delta \mathrm{TC}$ & $\Delta \mathrm{TSp}$ & $\triangle \mathrm{CPA}$ & $\mathrm{L}$ & $\mathrm{D}$ & $\mathrm{V}$ & Sea state \\
\hline \multicolumn{9}{|c|}{ Parallel courses - overtaking } \\
\hline 1 & $2 \mathrm{~V}$ & No & Yes & Yes & 289 & $16.8-14.5 / 31.1-26.9$ & $0 / 0$ & 3 \\
\hline 2 & $3 \mathrm{~V}$ & No & Yes & Yes & 290 & $6.7-3.4 / 12.4-6.3$ & $0 / 0$ & 2 \\
\hline 3 & $4 \mathrm{~V}$ & $\overline{\text { Yes }}$ & Yes & No & 180 & $19.8-19.6 / 36.7-36.3$ & $11.1 / 5.7$ & 4 \\
\hline 4 & $6 \mathrm{~V}$ & Yes & Yes & $\overline{\text { No }}$ & 225 & 16.8-16.4 / 31.1-30.4 & $10.8 / 5.6$ & 7 \\
\hline 5 & $7 \mathrm{~L}$ & Yes & Yes & No & 225 & 14.1-13.2 / 26.1-24.4 & $12.0 / 6.2$ & 3 \\
\hline 6 & 9L & Yes & No & $\overline{\text { Yes }}$ & 274 & $2.0-1.8 / 3.7-3.3$ & $14.0 / 7.2$ & 4 \\
\hline 7 & $11 \mathrm{~L}$ & Yes & $\overline{\text { Yes }}$ & No & 295 & $12.5-10.6$ / 23.2-19.6 & $11.5 / 5.9$ & 2 \\
\hline 8 & $12 \mathrm{~L}$ & No & No & No & 182 & $8.7-8.2 / 16.1-15.2$ & $15.5 / 8.0$ & 2 \\
\hline 9 & $17 \mathrm{M}$ & No & Yes & Yes & 207 & 18.9-15.8 / 35.0-29.3 & $0.5 / 0.3$ & 1 \\
\hline 10 & $21 \mathrm{M}$ & $\overline{\mathrm{Yes}}$ & Yes & No & 179 & 17.1-17.0 / 31.7-31.5 & $10.8 / 5.6$ & 6 \\
\hline 11 & $25 \mathrm{M}$ & Yes & Yes & No & 185 & 18.7-18.4 / 34.6-34.1 & $11.7 / 6.0$ & 1 \\
\hline 12 & $28 \mathrm{M}$ & Yes & Yes & No & 236 & $15.0-14.8$ / 27.8-27.4 & $14.6 / 7.5$ & 2 \\
\hline 13 & $37 \mathrm{M}$ & No & Yes & $\overline{\text { Yes }}$ & 289 & $9.4-3.5 / 17.4-6.5$ & $9.0 / 4.6$ & 5 \\
\hline 14 & $38 \mathrm{M}$ & $\overline{\mathrm{Yes}}$ & No & No & 299 & $20.4-18.9$ / 37.8-35.0 & $17.0 / 8.7$ & 1 \\
\hline 15 & $40 \mathrm{M}$ & Yes & No & $\overline{Y e s}$ & 261 & $24.4-23.8$ / 45.2-44.11 & $8.2 / 9.4$ & 1 \\
\hline 16 & $44 \mathrm{~S}$ & Yes & Yes & $\underline{\text { No }}$ & 190 & $5.7-4.7 / 10.6-8.7$ & $13.0 / 6.7$ & 4 \\
\hline 17 & $50 S$ & Yes & Yes & No & 145 & $4.8-4.5 / 8.9-8.3$ & $12.2 / 6.3$ & 3 \\
\hline 18 & $60 \mathrm{~S}$ & No & Yes & $\overline{Y e s}$ & 240 & $4.1-2.1 / 7.6-3.9$ & $1.6 / 0.8$ & 4 \\
\hline \multicolumn{9}{|c|}{ Reciprocal courses } \\
\hline 19 & $64 \mathrm{~L}$ & Yes & Yes & No & 177 & $13.1-6.6 / 24.3-12.2$ & $10.0 / 5.1$ & 5 \\
\hline 20 & $67 \mathrm{~L}$ & Yes & No & No & 333 & $12.5 / 23.2$ & $14.1 / 7.3$ & 4 \\
\hline 21 & $70 \mathrm{~L}$ & Yes & $\overline{\text { No }}$ & $\overline{Y e s}$ & 180 & $11.6-5.3 / 21.5-9.8$ & $11.1 / 5.7$ & 3 \\
\hline 22 & $81 \mathrm{M}$ & Yes & No & No & 127 & $14.2-10.6$ / 26.3-19.6 & $11.3 / 5.8$ & 3 \\
\hline 23 & $89 \mathrm{M}$ & No & Yes & Yes & 122 & $6.2-4.6 / 11.5-8.5$ & $13.5 / 6.9$ & 1 \\
\hline 24 & $90 \mathrm{M}$ & $\overline{\text { No }}$ & Yes & Yes & 115 & $11.0-4.2$ / 20.4-7.8 & $9.8 / 5.0$ & 2 \\
\hline 25 & $97 \mathrm{~S}$ & $\overline{\text { Yes }}$ & Yes & No & 112 & $9.1-5.3 / 16.9-9.8$ & $14.2 / 7.3$ & 2 \\
\hline 26 & $102 S$ & Yes & No & Yes & 102 & 6.3-1.7 / 11.7-3.1 & $10.2 / 5.2$ & 4 \\
\hline 27 & $105 S$ & No & Yes & Yes & 184 & 7.4-2.7 / 13.7-5.0 & $10.2 / 5.2$ & 3 \\
\hline \multicolumn{9}{|c|}{ Crossing courses } \\
\hline 28 & $118 \mathrm{~L}$ & Yes & Yes & No & 199 & 16.7-12.8 / 30.9-23.7 & $14.4 / 7.4$ & 2 \\
\hline 29 & $123 \mathrm{M}$ & Yes & Yes & No & 109 & 17.1-16.3 / 31.7-30.2 & $13.4 / 6.9$ & 5 \\
\hline 30 & $138 \mathrm{M}$ & No & No & Yes & 200 & 15.9-12.8 / 29.4-23.7 & $12.9 / 6.6$ & 1 \\
\hline 31 & $139 \mathrm{M}$ & Yes & No & Yes & 254 & 9.5-7.5 / 17.6-13.9 & 7.9 / 4.1 & 1 \\
\hline 32 & $140 \mathrm{M}$ & No & Yes & $\underline{\text { No }}$ & 179 & 21.8-20.5 / 40.4-38.0 & 7.2 / 3.7 & 2 \\
\hline 33 & $141 \mathrm{M}$ & No & Yes & No & 199 & 6.1-5.1 / 11.3-9.4 & $2.2 / 1.1$ & 7 \\
\hline 34 & $142 \mathrm{M}$ & No & No & No & 354 & 21.2-19.5 / 39.3-36.1 & $16.4 / 8.4$ & 6 \\
\hline 35 & $144 \mathrm{M}$ & No & Yes & No & 190 & 18.7-15.7 34.6-29.1 & $10.7 / 5.5$ & 6 \\
\hline 36 & $145 \mathrm{M}$ & No & No & No & 148 & $24.3-18.3$ / 45.0-33.9 & $10.8 / 5.6$ & 5 \\
\hline 37 & $146 \mathrm{M}$ & Yes & $\underline{\text { No }}$ & Yes & 108 & $18.9-15.6$ / 35.0-28.9 & $11.3 / 5.8$ & 5 \\
\hline 38 & $147 \mathrm{M}$ & No & Yes & No & 292 & 20.0-19.1 / 37.0-35.4 & $10.6 / 5.5$ & 5 \\
\hline 39 & $158 \mathrm{~S}$ & No & Yes & Yes & 96 & $2.9-2.3 / 5.4-4.3$ & $15.6 / 8.0$ & 3 \\
\hline 40 & $159 \mathrm{~S}$ & Yes & Yes & No & 117 & 16.6-15.9 / 30.7-29.4 & $12.4 / 6.4$ & 2 \\
\hline \multicolumn{9}{|c|}{ Both ships at anchor } \\
\hline 41 & $162 \mathrm{M}$ & - & - & No & 169 & $1.2 / 2.2$ & $0 / 0$ & 3 \\
\hline 42 & $163 \mathrm{M}$ & - & - & $\underline{\text { No }}$ & 183 & $1.7 / 3.2$ & $0 / 0$ & 3 \\
\hline 43 & $164 \mathrm{M}$ & - & - & No & 274 & $1.0 / 1.8$ & $0 / 0$ & 3 \\
\hline
\end{tabular}

Abbreviations used in Table 8 mean:

$\mathrm{D}$ - the distance between the ships (own and observed) during the measurement;

$\mathrm{L}$ - the length of the observed vessel presented on the web-site;

No - the differences in the indications of average value are greater than errors defined in IMO resolution and IEC standard;

Noms - number of meeting situation described in the Table 3;

Sea state - the state of the sea during the measurements expressed in degrees of the Douglas scale;

$\mathrm{V}$ - the mean value of the true speed of the observed ship;

Yes - the differences in the indications of average value are less than the errors defined in IMO resolution and IEC standard; and

$\overline{\triangle T C}, \triangle \mathrm{TSp}, \triangle \mathrm{CPA}$ - differences in the indications of average values of true course (TC), true speed (TSp) and CPA by ARPA and AIS. 
The information shown in Table 8 is summarized in Table 9.

Table 9. The number of measurement series with the differences in the indications of the average values of particular parameters by ARPA and AIS exceeding the error values specified in the IMO resolution and the IEC standard [own study]

\begin{tabular}{lllll}
\hline Parameter & \multicolumn{4}{l}{ Number of measurement series } \\
\hline TC & 1 & 2 & 3 & $\Sigma \mathrm{T}$ \\
TSp & 5 & 3 & 1 & 9 \\
TC \& TSp & 2 & 2 & 2 & 6 \\
CPA & 0 & 0 & 1 & 1 \\
CPA \& TC & 9 & 2 & 3 & 14 \\
CPA \& TSp & 0 & 0 & 4 & 4 \\
All data & 1 & 2 & 0 & 3 \\
$\Sigma$ T & 1 & 0 & 2 & 3 \\
& 18 & 9 & 13 & 40
\end{tabular}

Abbreviations used in Table 9 mean:

- 1,23 see Table 6; and

- $\Sigma \mathrm{T}$ - total number of measurement series with differences in the indications of the average values of particular parameters by ARPA and AIS exceeding the error values specified in the IMO resolution and the IEC standard.

The data presented in Table 8 show the dependence of the differences in AIS and ARPA indications on the true speed of the observed and tracked vessel and on the type of meeting situation. No other correlations could be found. The next Table
10 shows the relationship between the differences in AIS and ARPA indications and the errors in determining the average values of individual parameters by these devices.

Table 10. The number of measurement series in which the average value of the true course, true speed or CPA of the observed object indicated by ARPA and / or AIS had the error greater than its limit defined in the IMO resolution and IEC standard (for meeting situation in which differences in the indications of average true course, true speed or CPA by ARPA and AIS were greater than defined by international regulations [own study]

The average value of the The number of measurement series parameter presented with too in which the average value had too great error by great error

\begin{tabular}{|c|c|c|c|c|}
\hline I dy & TC & TSp & CPA & $\Sigma_{\mathrm{T}}$ \\
\hline AIS \& ARPA & 9 & 5 & 21 & 35 \\
\hline ARPA & 3 & 7 & 1 & 11 \\
\hline AIS & 0 & 0 & 0 & 0 \\
\hline $\begin{array}{l}\text { Mean value without too } \\
\text { great error }\end{array}$ & 5 & 1 & 2 & 8 \\
\hline$\Sigma_{\mathrm{T}}$ & 17 & 13 & 24 & 54 \\
\hline
\end{tabular}

Abbreviations as in the table 9.

The next table provides information on the state of the sea during the measurement series in which errors of the true course, true speed and / or CPA indications by ARPA and AIS on board equipment exceeded the values shown in Table 1.

Table 11. The state of the sea during the measurement series in which errors of the true course, true speed and / or CPA indications by ARPA and AIS on board equipment exceeded the values shown in Table 1 (for 95\% probability figures, excluding both ships at anchor) [own study]

\begin{tabular}{|c|c|c|c|c|c|c|c|c|c|}
\hline \multirow{3}{*}{$\begin{array}{l}\text { Data with errors exceeding the } \\
\text { values shown in Table } 1\end{array}$} & \multicolumn{8}{|c|}{ State of the sea expressed in degrees of the Douglas scale } & \multirow{2}{*}{$\Sigma$} \\
\hline & 1 & 2 & 3 & 4 & 5 & 6 & 7 & 8 & \\
\hline & \multicolumn{9}{|c|}{ ARPA indications } \\
\hline TC & 1 & 1 & - & - & - & - & - & - & 2 \\
\hline TSp & 4 & 7 & 13 & 5 & 7 & 1 & 1 & & 38 \\
\hline TC \& TSp & 2 & 1 & 2 & 1 & - & 1 & - & 1 & 8 \\
\hline $\mathrm{CPA}$ & 1 & 4 & 7 & 3 & - & - & - & - & 15 \\
\hline CPA \& TC & 1 & 2 & 2 & 1 & 2 & - & - & - & 8 \\
\hline CPA \& TSp & 4 & 5 & 2 & 2 & 1 & 3 & - & - & 17 \\
\hline All data & 4 & 5 & 7 & 2 & 6 & 3 & 3 & - & 30 \\
\hline$\Sigma / \Sigma_{\mathrm{T}}$ & $17 / 20$ & $25 / 35$ & $33 / 42$ & $14 / 26$ & $16 / 23$ & $8 / 9$ & $4 / 5$ & $1 / 1$ & $118 / 161$ \\
\hline \multicolumn{10}{|c|}{ AIS indications } \\
\hline TC & 1 & 2 & 6 & 1 & 1 & - & 1 & - & 12 \\
\hline TSp & 2 & - & 1 & 1 & 2 & - & - & - & 6 \\
\hline TC \& TSp & - & - & - & - & 1 & - & - & - & 1 \\
\hline CPA & 5 & 9 & 9 & 5 & 3 & 3 & - & - & 34 \\
\hline CPA \& TC & 1 & - & 2 & 1 & 3 & 1 & 1 & - & 9 \\
\hline CPA \& TSp & - & - & - & - & - & - & - & - & - \\
\hline All data & - & 1 & 1 & 1 & 1 & 2 & 2 & - & 8 \\
\hline$\Sigma / \Sigma_{\mathrm{T}}$ & $9 / 20$ & $12 / 35$ & $19 / 42$ & $9 / 26$ & $11 / 23$ & $6 / 9$ & $4 / 5$ & $-/ 1$ & $70 / 161$ \\
\hline
\end{tabular}

Collective information on the state of the sea during particular measurement series is presented in Table 12. Too few measurements in storm conditions makes it impossible to draw conclusions about the relationship between the accuracy of ARPA and AIS indications and the state of the sea.
Table 12. State of the sea expressed in degrees of the Douglas scale during the measurement series (including both ships at anchor) [own study]

\begin{tabular}{llllllll}
\hline $\begin{array}{c}\text { State of the sea 1 } \\
\text { (Douglas scale) }\end{array}$ & 2 & 3 & 4 & 5 & 6 & 7 & 8 \\
\hline $\begin{array}{c}\text { Number of 20 } \\
\text { measurement } \\
\text { series }\end{array}$ & 35 & $46^{\times}$ & 26 & 23 & 9 & 5 & 1 \\
\end{tabular}

$\bar{x}$ - including four measurement series, during which both ships were anchored 


\section{FINAL CONCLUSIONS}

Measurements described in this paper were carried out on ten different merchant ships using popular, often used AIS and radar on board equipment but produced by four manufacturers only. Due to that and due to the limited number of conducted tests (161 series of measurements while both ships are underway and 4 when they are at anchors), it is impossible to formulate on their basis general conclusions about the stability and accuracy of the AIS and ARPA indications and their dependence on hydro meteorological conditions. Nevertheless, the performed measurements allow for the formulation of some remarks on these topics.

Table 6 shows that the accuracy of ARPA indications does not depend on the type of ships meeting situation. ARPA presented all or part of data with the accuracy lower than defined by international recommendations and standard (presented in Table 1) in $73 \%$ of the measurement series (118 out of 161). It mainly had a problem with the accuracy of the presentation of the true speed of the tracked vessel (in $24 \%$ of meeting situations (38 out of 161)). All ARPA data had too great error in $19 \%$ of observations (30 out of 161), CPA, or CPA and true course, or CPA and true speed in $25 \%$ (40 out of 161). Generally, ARPA reported a CPA value with an error greater than 0.3 nautical miles in $43 \%$ of meetings (70 out of 161 ). AIS had problems mainly with the accurate presentation of CPA value (in $32 \%$ of measurement series (51 out of $161)$ ), especially in the case of overtaking ships on parallel courses (in $49 \%$ percent of observations (30 out of 61 measurement series)).

The data presented in Table 7 show that there was no correlation between the inaccuracies in the AIS and ARPA indications for the same object. Out of the total number of 30 measurement series in which ARPA incorrectly presented all recorded data, AIS also showed all data with too great errors in only 5 series (in 9 series the inaccuracy was related to CPA value, in 8 series related to CPA and true course). In 15 meeting situations where ARPA had problems with accurately presenting only CPA values, AIS also incorrectly showed only CPA in 8 tests. In 38 tests, where ARPA showed insufficiently accurate information about the true speed of the tracked vessel, AIS had problems with the accurate presentation of its true speed in 5 tests only and in 29 tests presented all data of this vessel with the required accuracy.

The tests carried out have shown that both ARPA and AIS can indicate distances between two ships at anchors unstable and with significant inaccuracies.

A separate issue are the differences between the AIS and ARPA indications of the average values of the observed and tracked object true course, true speed and CPA. In a significant number of tests (in $24 \%$ of the measurement series), they exceeded the values of the presentation errors defined in the IMO Resolution MSC.192(79) and IEC Standard 61993-2. It should be noted that in all these cases, the AIS presented the mean values of individual parameters with acceptable errors. It is interesting that in 8 cases of the discussed differences in the AIS and ARPA indications, both devices showed average values of all parameters with errors smaller than those defined in the international regulations.

The results of the measurements presented in Table 11 do not show a clear relationship between the accuracy of the data presented by AIS and ARPA and the current level of disturbances from the sea surface. The reason for this may be the lack of a comparable number of measurements carried out in individual sea states expressed in the Douglas scale and too small number of tests conducted in storm weather condition (Table 12)

The comments presented in this article should be taken into account when using ARPA and AIS as technical means of observation for anti-collision purposes. They show that the indications of both devices in real conditions may be unstable and have errors greater than those specified in the IMO Resolution MSC.192(79) and IEC Standard 61993-2. Therefore, users should not rely on the instantaneous digital data values of the other vessel presented by ARPA and AIS.

\section{REFERENCES}

1. IEC Standard 61993-2 ED 3 "Maritime navigation and radiocommunication equipment and systems Automatic identification systems (AIS) - Part 2: Class A shipborne equipment of the automatic identification system (AIS) - Operational and performance requirements, methods of test and required test results", IEC, Geneva 2017.

2. IEC Standard 62388 "Maritime navigation and radiocommunication equipment and systems - Shipborne radar - Performance requirements, methods of testing and required test results", IEC, Geneva 2013.

3. IMO Resolution A.1106(29) "Revised guidelines for the onboard use of shipborne automatic identification system (AIS)", IMO, London 2015.

4. IMO Resolution MSC.192(79) "Adoption of the revised performance standards for radar equipment", IMO, London 2004.

5. Kalamon M., "AIS as an additional means of observing and assessing collision risk", engineering thesis, Gdynia Maritime University, Gdynia 2017.

6. Nawrocki M., "Experimental research on accuracy of the closest point of approach indication by AIS and radar equipment", engineering thesis, Gdynia Maritime University, Gdynia 2019.

7. Orzeszko K. R., "Comparative analysis of the accuracy and stability of AIS and ARPA indications of approaching parameters", engineering thesis, Gdynia Maritime University, Gdynia 2019.

8. Piekarska M. K., "Experimental research on the accuracy and stability of indications of the opposite vessel's motion vectors by AIS and the radar equipment", engineering thesis, Gdynia Maritime University, Gdynia 2019.

9. Ręgocki M., "Experimental research on the accuracy of radar tracking", engineering thesis, Gdynia Maritime University, Gdynia 2019.

10. Surkov G., "Experimental research of relative and true motion indications using ARPA and AIS devices", engineering thesis, Gdynia Maritime University, Gdynia 2020.

11. Trochimiak A., "Accuracy of indication of the Closest Point of Approach as a function of the tracked object true motion vector stability", engineering thesis, Gdynia Maritime University, Gdynia 2020. 
12. Wawruch R., "Use of automatic identification system as a source of information to avoid ships' collisions at sea", Management Perspective for Transport Telematics 2018, 18th International Conference on Transport System Telematics, TST 2018, Krakow, Poland, March 20-23, 2018, Selected Papers, Ed. Jerzy Mikulski, Communications in Computer and Information Science Book Series 7899 (CCIS, volume 897), Springer International Publishing, 2018, pp.411-425.

13. Wawruch R., "Tests of the Accuracy of Indications by ARPA and AIS of the Opposite Vessel True Course, True Speed and $\mathrm{CPA}^{\prime \prime}$, The 19th International Radar Symposium "IRS 2018", June 20-22, 2018, Bonn, 2018, Proceedings, DGON, Bonn 2018 (CD).

14. Wawruch R., "Comparative study of the accuracy of AIS and ARPA indications. Part 1. Accuracy of the CPA indications", The International Journal on Marine
Navigation and Safety of Sea Transportation "TransNav", Vol. 12, No 3, September 2018, pp. 439-443.

15. Wawruch R., "Comparative study of the accuracy of AIS and ARPA indications. Part 2. Accuracy of the opposite vessel true course and true speed indication", The International Journal on Marine Navigation and Safety of Sea Transportation "TransNav", Vol. 12, No 4, December 2018, pp. 1-4.

16. Wesołowski J., "Comparative analysis of the accuracy of data on the relative and true motion parameters of an opposite ship presented by ARPA and AIS", engineering thesis, Gdynia Maritime University, Gdynia 2016.

17. Wilczyński M., "Comparative analysis of AIS and ARPA tracking accuracy", engineering thesis, Gdynia Maritime University, Gdynia 2015.

18. Wilk I. D., "Accuracy of distance and true motion vector indications by radar tracking systems", engineering thesis, Gdynia Maritime University, Gdynia 2020. 